\title{
PROPERTIES OF SOME FAMILIES OF HYPERGEOMETRIC ORTHOGONAL POLYNOMIALS IN SEVERAL VARIABLES
}

\author{
J. F. VAN DIEJEN
}

\begin{abstract}
Limiting cases are studied of the Koornwinder-Macdonald multivariable generalization of the Askey-Wilson polynomials. We recover recently and not so recently introduced families of hypergeometric orthogonal polynomials in several variables consisting of multivariable Wilson, continuous Hahn and Jacobi type polynomials, respectively. For each class of polynomials we provide systems of difference (or differential) equations, recurrence relations, and expressions for the (squared) norms of the polynomials in question.
\end{abstract}

\section{INTRODUCTION}

It is over a decade ago that Askey and Wilson released their famous memoir [AW2], in which they introduced a four-parameter family of basic hypergeometric polynomials nowadays commonly referred to as the Askey-Wilson polynomials [GR]. These polynomials, which are defined explicitly in terms of a terminating ${ }_{4} \phi_{3}$ series, have been shown to exhibit a number of interesting properties. Among other things, it was demonstrated that they satisfy a second order difference equation, a threeterm recurrence relation, and that - in a suitable parameter regime - they constitute an orthogonal system with respect to an explicitly given positive weight function with support on a finite interval (or on the unit circle, depending on how the coordinates are chosen).

Many (basic) hypergeometric orthogonal polynomials studied in the literature arise as special (limiting) cases of the Askey-Wilson polynomials and have been collected in the so-called $(q-)$ Askey scheme $[\mathrm{AW} 2, \mathrm{KS}]$. For instance, if the step size parameter of the difference equation is scaled to zero, then the Askey-Wilson polynomials go over into Jacobi polynomials: well-known classical hypergeometric orthogonal polynomials satisfying a second order differential equation instead of a difference equation. One may also consider the transition from orthogonal polynomials on a finite interval to orthogonal polynomials on a (semi-)infinite interval. This way one arrives at Wilson polynomials [W] (semi-infinite interval) and at continuous Hahn polynomials [AW1, AtSu, A] (infinite interval).

Received by the editors April 8, 1996 and, in revised form, November 25, 1996.

1991 Mathematics Subject Classification. Primary 33C50; Secondary 33D45.

Key words and phrases. Orthogonal polynomials in several variables, difference and differential equations, recurrence relations, orthonormalization constants, Selberg type integrals, quantum integrable $n$-particle systems.

Work supported by the Japan Society for the Promotion of Science (JSPS) and by a Monbusho Grant-in-Aid. 
The purpose of the present paper is to generalize this state of affairs from one to several variables. Our starting point is a recently introduced multivariable generalization of the Askey-Wilson polynomials, found for special parameters by Macdonald [M2] and in full generality (involving five parameters) by Koornwinder [K2]. By means of limiting transitions similar to those in the one-variable case, we arrive at multivariable Jacobi polynomials [V, De, H] (see also [BO] and the references therein) and at multivariable Wilson and continuous Hahn polynomials [D3].

The $(q-)$ Askey scheme involves many more limits and special cases of the AskeyWilson polynomials than those described above. For instance, one also considers transitions from certain polynomials in the scheme to similar polynomials with less parameters and transitions from polynomials with a continuous orthogonality measure to polynomials with a discrete orthogonality measure. Such transitions (or rather their multivariable analogues) will not be considered here. We refer instead to [D1, Sec. 5.2] for the transition from multivariable Askey-Wilson polynomials to Macdonald's $q$-Jack polynomials (i.e., multivariable $q$-ultraspherical polynomials) [M4] (as an example of a limit leading to similar polynomials but with less parameters), and to [SK] for the transition from multivariable Askey-Wilson polynomials to multivariable big and little $q$-Jacobi polynomials [S1] (as an example of a limit leading to multivariable polynomials with a discrete orthogonality measure).

Whenever one is dealing with orthogonal polynomials an important question arises as to the explicit computation of the normalization constants converting the polynomials into an orthonormal system. For Jacobi polynomials calculating the orthonormalization constants boils down to the evaluation of (standard) beta integrals, whereas Askey-Wilson polynomials give rise to $q$-beta integrals. In the case of several variables one has to deal with Selberg type integrals (Jacobi case) and $q$-Selberg type integrals (Askey-Wilson case), respectively. For these multiple integrals explicit evaluations have been conjectured by Macdonald, which were recently checked using techniques involving so-called shift operators [Op1, HS, C3, M5]. (Roughly speaking these shift operators allow one to relate the values of the $(q-)$ Selberg integral for different values of the parameters separated by unit shifts; the integral can then be solved, first for nonnegative integer-valued parameters by shifting the parameters to zero, in which case the integrand becomes trivial, and then for arbitrary nonnegative parameters using an analyticity argument (viz., Carlson's theorem).)

Very recently, the author observed that Koornwinder's second order difference equation for the multivariable Askey-Wilson polynomials may be extended to a system of $n$ (= number of variables) independent difference equations [D1] and that the polynomials also satisfy a system of $n$ independent recurrence relations [D5]. (To date a complete proof for these recurrence relations is available only for a selfdual four-parameter subfamily of the five-parameter multivariable Askey-Wilson polynomials.) It turns out that the recurrence relations, combined with the known evaluation for the norm of the unit polynomial (i.e., the constant term integral) [Gu1, Ka], may also be used to verify Macdonald's formulas for the orthonormalization constants of the multivariable Askey-Wilson polynomials [D5]. Below, we will use these results to arrive at systems of difference (or differential) equations, recurrence relations and expressions for the orthonormalization constants, for all three limiting cases of the multivariable Askey-Wilson polynomials considered in this paper (Wilson, continuous Hahn and Jacobi type). 
We would like to emphasize that much of the material presented here admits a physical interpretation in terms of Calogero-Sutherland type exactly solvable quantum $n$-particle models related to classical root systems $[\mathrm{OP}]$ or their Ruijsenaars type difference versions [R1, R2, D2]. The point is that the second order differential equation for the multivariable Jacobi polynomials may be seen as the eigenvalue equation for a trigonometric quantum Calogero-Sutherland system related to the root system $B C_{n}[\mathrm{OP}]$. From this viewpoint the second order difference equation for the multivariable Askey-Wilson polynomials corresponds to the eigenvalue equation for a Ruijsenaars type difference version of the $B C_{n}$-type quantum CalogeroSutherland system [D4]. The transitions to the multivariable continuous Hahn and Wilson polynomials amount to rational limits leading to (the eigenfunctions of) similar difference versions of the $A_{n-1}$-type rational Calogero model with harmonic term (continuous Hahn case) and its $B(C)_{n}$-type counterpart (Wilson case) [D3]. For further details regarding these connections with the Calogero-Sutherland and Ruijsenaars type quantum integrable $n$-particle systems the reader is referred to [D2, D3, D4].

The material is organized as follows. First we define our families of multivariable (basic) hypergeometric polynomials in Section 2 and recall their second order difference equation (Askey-Wilson, Wilson, continuous Hahn type) or second order differential equation (Jacobi type) in Section 3. Next, in Section 4, limit transitions from the Askey-Wilson type family to the Wilson, continuous Hahn and Jacobi type families are discussed. We study the behavior of our recently introduced systems of difference equations and recurrence relations for the multivariable Askey-Wilson type polynomials with respect to these limits in Sections 5 and 6 , respectively. The recurrence relations for the Wilson, continuous Hahn and Jacobi type polynomials thus obtained in Section 6 are then employed in Section 7 to derive explicit expressions for the (squared) norms of the corresponding polynomials in terms of the (squared) norm of the unit polynomial.

\section{Multivariable (BAsic) Hypergeometric POlynomials}

In this section multivariable versions of some orthogonal families of (basic) hypergeometric polynomials are characterized. The general idea of the construction (which is standard, see e.g. [V, M2, K2, SK]) is to start with an algebra of (symmetric) polynomials $\mathcal{H}$ spanned by a basis of (symmetric) monomials $\left\{m_{\lambda}\right\}_{\lambda \in \Lambda}$, with the set $\Lambda$ labeling the basis elements being partially ordered in such a way that for all $\lambda \in \Lambda$ the subspaces $\mathcal{H}_{\lambda} \equiv \operatorname{Span}\left\{m_{\mu}\right\}_{\mu \in \Lambda, \mu \leq \lambda}$ are finite-dimensional. It is furthermore assumed that the space $\mathcal{H}$ is endowed with an $L^{2}$ inner product $\langle\cdot, \cdot\rangle_{\Delta}$ characterized by a certain weight function $\Delta$. To such a configuration we associate a basis $\left\{p_{\lambda}\right\}_{\lambda \in \Lambda}$ of $\mathcal{H}$ consisting of the polynomials $p_{\lambda}, \lambda \in \Lambda$, determined (uniquely) by the two conditions

$$
\begin{aligned}
& \text { i. } p_{\lambda}=m_{\lambda}+\sum_{\mu \in \Lambda, \mu<\lambda} c_{\lambda, \mu} m_{\mu}, \quad c_{\lambda, \mu} \in \mathbb{C} \text {; } \\
& \text { ii. }\left\langle p_{\lambda}, m_{\mu}\right\rangle_{\Delta}=0 \text { if } \mu<\lambda .
\end{aligned}
$$

In other words, the polynomial $p_{\lambda}$ consists of the monomial $m_{\lambda}$ minus its orthogonal projection with respect to the inner product $\langle\cdot, \cdot\rangle_{\Delta}$ onto the finite-dimensional subspace $\operatorname{Span}\left\{m_{\mu}\right\}_{\mu \in \Lambda, \mu<\lambda}$. By varying the concrete choices for the space $\mathcal{H}$, the basis $\left\{m_{\lambda}\right\}_{\lambda \in \Lambda}$ and the inner product $\langle\cdot, \cdot\rangle_{\Delta}$, we recover certain (previously introduced) multivariable generalizations of the Askey-Wilson, Wilson, continuous Hahn 
and Jacobi polynomials. Below we will specify the relevant data determining these families. The fact that in the case of one variable the corresponding polynomials $p_{\lambda}$ indeed reduce to the well-known one-variable polynomials studied extensively in the literature is immediate from the weight function. The normalization for the polynomials is determined by the fact that (by definition) $p_{\lambda}$ is monic in the sense that the coefficient of the leading monomial $m_{\lambda}$ in $p_{\lambda}$ is equal to one.

It turns out that in all of our cases the basis $\left\{m_{\lambda}\right\}_{\lambda \in \Lambda}$ can be conveniently expressed in terms of the monomial symmetric functions

$$
m_{\text {sym }, \lambda}\left(z_{1}, \ldots, z_{n}\right)=\sum_{\mu \in S_{n}(\lambda)} z_{1}^{\mu_{1}} \cdots z_{n}^{\mu_{n}}, \quad \lambda \in \Lambda,
$$

where

$$
\Lambda=\left\{\lambda \in \mathbb{Z}^{n} \mid \lambda_{1} \geq \lambda_{2} \geq \cdots \geq \lambda_{n} \geq 0\right\} .
$$

In (2.1) the summation is meant over the orbit of $\lambda$ under the action of the permutation group $S_{n}$ (acting on the vector components $\lambda_{1}, \ldots, \lambda_{n}$ ). As partial order of the integral cone $\Lambda(2.2)$ we will always take the dominance type order defined by

$$
\mu \leq \lambda \quad \text { iff } \quad \sum_{j=1}^{m} \mu_{j} \leq \sum_{j=1}^{m} \lambda_{j} \text { for } m=1, \ldots, n
$$

(and $\mu<\lambda$ iff $\mu \leq \lambda$ and $\mu \neq \lambda$ ).

Note: In order to avoid confusion between the various families we will often equip the polynomials and other objects of interest with the superscripts 'AW', 'W', 'cH' or ' $\mathrm{J}$ ' to indicate Askey-Wilson, Wilson, continuous Hahn or Jacobi type polynomials, respectively. Sometimes, however, these superscripts will be suppressed when discussing more general properties of the polynomials that hold simultaneously for all families.

2.1. Askey-Wilson type. To arrive at multivariable Askey-Wilson type polynomials one considers a space $\mathcal{H}^{A W}$ consisting of even and permutation invariant trigonometric polynomials. Specifically, the space $\mathcal{H}^{A W}$ is spanned by the monomials

$$
m_{\lambda}^{A W}(x)=m_{\text {sym }, \lambda}\left(e^{i \alpha x_{1}}+e^{-i \alpha x_{1}}, \cdots, e^{i \alpha x_{n}}+e^{-i \alpha x_{n}}\right), \quad \lambda \in \Lambda
$$

(with $\Lambda$ given by (2.2)). The relevant inner product on $\mathcal{H}^{A W}$ is determined by

$$
\begin{aligned}
& \left\langle m_{\lambda}^{A W}, m_{\mu}^{A W}\right\rangle_{\Delta A W} \\
& \quad=\left(\frac{\alpha}{2 \pi}\right)^{n} \int_{-\pi / \alpha}^{\pi / \alpha} \cdots \int_{-\pi / \alpha}^{\pi / \alpha} m_{\lambda}^{A W}(x) \overline{m_{\mu}^{A W}(x)} \Delta^{A W}(x) d x_{1} \cdots d x_{n},
\end{aligned}
$$

with the weight function reading

$$
\begin{aligned}
\Delta^{A W}(x)= & \prod_{\substack{1 \leq j<k \leq n \\
\varepsilon_{1}, \varepsilon_{2}= \pm 1}} \frac{\left(e^{i \alpha\left(\varepsilon_{1} x_{j}+\varepsilon_{2} x_{k}\right)} ; q\right)_{\infty}}{\left(t e^{i \alpha\left(\varepsilon_{1} x_{j}+\varepsilon_{2} x_{k}\right)} ; q\right)_{\infty}} \\
& \times \prod_{\substack{1 \leq j \leq n \\
\varepsilon= \pm 1}} \frac{\left(e^{2 i \alpha \varepsilon x_{j}} ; q\right)_{\infty}}{\left(t_{0} e^{i \alpha \varepsilon x_{j}}, t_{1} e^{i \alpha \varepsilon x_{j}}, t_{2} e^{i \alpha \varepsilon x_{j}}, t_{3} e^{i \alpha \varepsilon x_{j}} ; q\right)_{\infty}} .
\end{aligned}
$$


Here $(a ; q)_{\infty} \equiv \prod_{m=0}^{\infty}\left(1-a q^{m}\right),\left(a_{1}, \ldots, a_{r} ; q\right)_{\infty} \equiv\left(a_{1} ; q\right)_{\infty} \cdots\left(a_{r} ; q\right)_{\infty}$ and the parameters are assumed to satisfy the constraints

$$
\alpha>0, \quad 0<q<1, \quad-1<t \leq 1, \quad\left|t_{r}\right| \leq 1 \quad(r=0,1,2,3),
$$

with possible non-real parameters $t_{r}$ occurring in complex conjugate pairs and pairwise products of the $t_{r}$ being $\neq 1$. For the weight function in (2.6) the polynomials $p_{\lambda}$ determined by conditions $i$. and $i$. (above) were introduced by Macdonald [M2] (for special parameters) and Koornwinder [K2] (for general parameters). In the special case of one variable $(n=1)$ these polynomials reduce to monic Askey-Wilson polynomials [AW2, KS]:

$$
\begin{aligned}
p_{l}^{A W}(x)= & \frac{\left(t_{0} t_{1}, t_{0} t_{2}, t_{0} t_{3} ; q\right)_{l}}{t_{0}^{l}\left(t_{0} t_{1} t_{2} t_{3} q^{l-1} ; q\right)_{l}} \\
& \times_{4} \phi_{3}\left(\begin{array}{c}
q^{-l}, t_{0} t_{1} t_{2} t_{3} q^{l-1}, t_{0} e^{i \alpha x}, t_{0} e^{-i \alpha x} \\
t_{0} t_{1}, t_{0} t_{2}, t_{0} t_{3}
\end{array} ;, q\right) .
\end{aligned}
$$

2.2. Wilson type. In the Wilson case the appropriate space $\mathcal{H}^{W}$ consists of even and permutation invariant polynomials and is spanned by the monomials

$$
m_{\lambda}^{W}(x)=m_{\text {sym }, \lambda}\left(x_{1}^{2}, \cdots, x_{n}^{2}\right), \quad \lambda \in \Lambda .
$$

The inner product on $\mathcal{H}^{W}$ is now determined by

$$
\left\langle m_{\lambda}^{W}, m_{\mu}^{W}\right\rangle_{\Delta^{W}}=\left(\frac{1}{2 \pi}\right)^{n} \int_{-\infty}^{\infty} \cdots \int_{-\infty}^{\infty} m_{\lambda}^{W}(x) \overline{m_{\mu}^{W}(x)} \Delta^{W}(x) d x_{1} \cdots d x_{n},
$$

with the weight function taken to be of the form

$$
\begin{aligned}
\Delta^{W}(x)= & \prod_{\substack{1 \leq j<k \leq n \\
\varepsilon_{1}, \varepsilon_{2}= \pm 1}} \frac{\Gamma\left(\nu+i\left(\varepsilon_{1} x_{j}+\varepsilon_{2} x_{k}\right)\right)}{\Gamma\left(i\left(\varepsilon_{1} x_{j}+\varepsilon_{2} x_{k}\right)\right)} \\
& \times \prod_{\substack{1 \leq j \leq n \\
\varepsilon= \pm 1}} \frac{\Gamma\left(\nu_{0}+i \varepsilon x_{j}\right) \Gamma\left(\nu_{1}+i \varepsilon x_{j}\right) \Gamma\left(\nu_{2}+i \varepsilon x_{j}\right) \Gamma\left(\nu_{3}+i \varepsilon x_{j}\right)}{\Gamma\left(2 i \varepsilon x_{j}\right)} .
\end{aligned}
$$

Here $\Gamma(\cdot)$ denotes the gamma function, and the parameters are such that

$$
\nu \geq 0, \quad \operatorname{Re}\left(\nu_{r}\right)>0 \quad(r=0,1,2,3),
$$

with possible non-real parameters $\nu_{r}$ occurring in complex conjugate pairs. For this weight function (and real parameters) the polynomials $p_{\lambda}$ were introduced in [D3]. In the case of one variable they reduce to monic Wilson polynomials [W, KS]

$$
\begin{aligned}
p_{l}^{W}(x)= & \frac{\left(\nu_{0}+\nu_{1}, \nu_{0}+\nu_{2}, \nu_{0}+\nu_{3}\right)_{l}}{(-1)^{l}\left(\nu_{0}+\nu_{1}+\nu_{2}+\nu_{3}+l-1\right)_{l}} \\
& \quad \times{ }_{4} F_{3}\left(\begin{array}{c}
-l, \nu_{0}+\nu_{1}+\nu_{2}+\nu_{3}+l-1, \nu_{0}+i x, \nu_{0}-i x \\
\nu_{0}+\nu_{1}, \nu_{0}+\nu_{2}, \nu_{0}+\nu_{3}
\end{array} ; 1\right) .
\end{aligned}
$$

2.3. Continuous Hahn type. The space $\mathcal{H}^{c H}$ is very similar to that of the Wilson case, but instead of only the even sector it now consists of all permutation invariant polynomials. The monomial basis for the space $\mathcal{H}^{c H}$ then becomes

$$
m_{\lambda}^{c H}(x)=m_{\text {sym }, \lambda}\left(x_{1}, \cdots, x_{n}\right), \quad \lambda \in \Lambda .
$$


The inner product is of the same form as for the Wilson case

$$
\left\langle m_{\lambda}^{c H}, m_{\mu}^{c H}\right\rangle_{\Delta^{c H}}=\left(\frac{1}{2 \pi}\right)^{n} \int_{-\infty}^{\infty} \cdots \int_{-\infty}^{\infty} m_{\lambda}^{c H}(x) \overline{m_{\mu}^{c H}(x)} \Delta^{c H}(x) d x_{1} \cdots d x_{n}
$$

but now with a weight function given by

$$
\begin{aligned}
\Delta^{c H}(x)= & \prod_{1 \leq j<k \leq n}\left(\frac{\Gamma\left(\nu+i\left(x_{j}-x_{k}\right)\right)}{\Gamma\left(i\left(x_{j}-x_{k}\right)\right)} \frac{\Gamma\left(\nu+i\left(x_{k}-x_{j}\right)\right)}{\Gamma\left(i\left(x_{k}-x_{j}\right)\right)}\right) \\
& \times \prod_{1 \leq j \leq n} \Gamma\left(\nu_{0}^{+}+i x_{j}\right) \Gamma\left(\nu_{1}^{+}+i x_{j}\right) \Gamma\left(\nu_{0}^{-}-i x_{j}\right) \Gamma\left(\nu_{1}^{-}-i x_{j}\right),
\end{aligned}
$$

where

$$
\nu \geq 0, \quad \operatorname{Re}\left(\nu_{0}^{ \pm}\right), \operatorname{Re}\left(\nu_{1}^{ \pm}\right)>0, \quad \nu_{0}^{-}=\overline{\nu_{0}^{+}}, \quad \nu_{1}^{-}=\overline{\nu_{1}^{+}} .
$$

(The condition $\nu_{r}^{-}=\bar{\nu}_{r}^{+}$is assumed in order to guarantee that the weight function $\Delta^{c H}(x)$ in (2.16) is real and positive; cf. also Remark $i$. below.) Just as in the case of Wilson type polynomials, the polynomials $p_{\lambda}^{c H}$ corresponding to the weight function (2.16) were introduced in [D3]. For $n=1$ they reduce to monic continuous Hahn polynomials [AW1, AtSu, A, KS]

$$
\begin{aligned}
p_{l}^{c H}(x)=\frac{i^{l}\left(\nu_{0}^{+}+\nu_{0}^{-}, \nu_{0}^{+}+\nu_{1}^{-}\right)_{l}}{\left(\nu_{0}^{+}+\nu_{0}^{-}+\nu_{1}^{+}+\nu_{1}^{-}+l-1\right)_{l}} & \\
& \times{ }_{3} F_{2}\left(\begin{array}{c}
-l, \nu_{0}^{+}+\nu_{0}^{-}+\nu_{1}^{+}+\nu_{1}^{-}+l-1, \nu_{0}^{+}+i x \\
\nu_{0}^{+}+\nu_{0}^{-}, \nu_{0}^{+}+\nu_{1}^{-}
\end{array}\right] .
\end{aligned}
$$

2.4. Jacobi type. The space $\mathcal{H}^{J}$ and the basis $\left\{m_{\lambda}^{J}\right\}_{\lambda \in \Lambda}$ are the same as for the Askey-Wilson type. The inner product is also of the form given there (cf. (2.5)), but the weight function gets replaced by

$$
\begin{aligned}
\Delta^{J}(x)= & \prod_{1 \leq j<k \leq n}\left|\sin \frac{\alpha}{2}\left(x_{j}+x_{k}\right) \sin \frac{\alpha}{2}\left(x_{j}-x_{k}\right)\right|^{2 \nu} \\
& \times \prod_{1 \leq j \leq n}\left|\sin \left(\frac{\alpha}{2} x_{j}\right)\right|^{2 \nu_{0}}\left|\cos \left(\frac{\alpha}{2} x_{j}\right)\right|^{2 \nu_{1}}
\end{aligned}
$$

with

$$
\alpha>0, \quad \nu \geq 0, \quad \nu_{0}, \nu_{1}>-1 / 2 .
$$

In this case the corresponding polynomials $p_{\lambda}$ were first introduced by Vretare [V] (see also [De, H, BO]). For $n=1$ they reduce to monic Jacobi polynomials [AbSt, KS]

$$
p_{l}^{J}(x)=\frac{2^{2 l}\left(\nu_{0}+1 / 2\right)_{l}}{\left(\nu_{0}+\nu_{1}+l\right)_{l}}{ }_{2} F_{1}\left(\begin{array}{c}
-l, \nu_{0}+\nu_{1}+l \\
\nu_{0}+1 / 2
\end{array} ; \sin ^{2}\left(\frac{\alpha x}{2}\right)\right) .
$$

Remarks. $i$. For the parameter regimes described above, the weight functions for the multivariable Askey-Wilson, Wilson, continuous Hahn and Jacobi polynomials are positive and continuous; moreover, the integral determining the corresponding inner product $\langle\cdot, \cdot\rangle_{\Delta}$ converges in absolute value. It is expected that (similarly to the known situation in the case of one variable, cf. [W, AW2, KS]) these orthogonality domains may be extended to a larger set of parameter values if one allows for a weight function with mixed continuous/discrete parts. For the multivariable 
Askey-Wilson case such an extension of the orthogonality domain to more general parameter values was recently considered by Stokman [S2].

ii. In the explicit formulas for the polynomials when $n=1$ we have used their standard representations in terms of terminating (basic) hypergeometric series [AW2, KS]

$$
\begin{aligned}
{ }_{r} F_{s}\left(\begin{array}{c}
a_{1}, \ldots, a_{r} \\
b_{1}, \ldots, b_{s}
\end{array} ; z\right) & =\sum_{k=0}^{\infty} \frac{\left(a_{1}, \ldots, a_{r}\right)_{k}}{\left(b_{1}, \ldots, b_{s}\right)_{k}} \frac{z^{k}}{k !}, \\
{ }_{s+1} \phi_{s}\left(\begin{array}{c}
a_{1}, \ldots, a_{s+1} \\
b_{1}, \ldots, b_{s}
\end{array} ; q, z\right) & =\sum_{k=0}^{\infty} \frac{\left(a_{1}, \ldots, a_{s+1} ; q\right)_{k}}{\left(b_{1}, \ldots, b_{s} ; q\right)_{k}} \frac{z^{k}}{(q ; q)_{k}},
\end{aligned}
$$

where we have used Pochhammer symbols and $q$-shifted factorials defined by

$$
\left(a_{1}, \ldots, a_{r}\right)_{k}=\left(a_{1}\right)_{k} \cdots\left(a_{r}\right)_{k}, \quad\left(a_{1}, \ldots, a_{r} ; q\right)_{k}=\left(a_{1} ; q\right)_{k} \cdots\left(a_{r} ; q\right)_{k}
$$

with $(a)_{0}=(a ; q)_{0}=1$ and

$$
(a)_{k}=a(a+1) \cdots(a+k-1), \quad(a ; q)_{k}=(1-a)(1-a q) \cdots\left(1-a q^{k-1}\right)
$$

for $k=1,2,3, \ldots$

\section{SECOND ORDER DifFERENCE OR DIFFERENTIAL EQUATIONS}

As it turns out, all families of polynomials $\left\{p_{\lambda}\right\}_{\lambda \in \Lambda}$ introduced in the previous section satisfy an eigenvalue equation of the form

$$
D p_{\lambda}=E_{\lambda} p_{\lambda}, \quad \lambda \in \Lambda
$$

where $D: \mathcal{H} \rightarrow \mathcal{H}$ denotes a certain second order difference operator (Askey-Wilson, Wilson and continuous Hahn case) or a second order differential operator (Jacobi case). Below we will list for each family the relevant operator $D$ together with its eigenvalues $E_{\lambda}, \lambda \in \Lambda$. In each case the proof that the polynomials $p_{\lambda}$ indeed satisfy the corresponding eigenvalue equations boils down to demonstrating that the operator $D: \mathcal{H} \rightarrow \mathcal{H}$ maps the finite-dimensional subspaces $\mathcal{H}_{\lambda}=\operatorname{Span}\left\{m_{\mu}\right\}_{\mu \in \Lambda, \mu \leq \lambda}$ into themselves (triangularity) and that it is symmetric with respect to the inner product $\langle\cdot, \cdot\rangle_{\Delta}$. In other words, one has to show that

Triangularity

$$
D m_{\lambda}=\sum_{\mu \in \Lambda, \mu \leq \lambda}[D]_{\lambda, \mu} m_{\mu}, \quad \text { with }[D]_{\lambda, \mu} \in \mathbb{C},
$$

and that

Symmetry

$$
\left\langle D m_{\lambda}, m_{\mu}\right\rangle_{\Delta}=\left\langle m_{\lambda}, D m_{\mu}\right\rangle_{\Delta} .
$$

It is immediate from these two properties and the definition of the polynomial $p_{\lambda}$ that $D p_{\lambda}$ lies in $\mathcal{H}_{\lambda}$ and is orthogonal with respect to $\langle\cdot, \cdot\rangle_{\Delta}$ to all monomials $m_{\mu}$, $\mu \in \Lambda$ with $\mu<\lambda$. But then comparison with the definition of $p_{\lambda}$ shows that $D p_{\lambda}$ must be proportional to $p_{\lambda}$, i.e., $p_{\lambda}$ is an eigenfunction of $D$. The corresponding eigenvalue $E_{\lambda}$ is obtained via an explicit computation of the diagonal matrix element $[D]_{\lambda, \lambda}$ in expansion (3.2).

For the Jacobi case a proof of the second order differential equation along the above lines was given by Vretare [V]. In the Askey-Wilson case the proof was given by Macdonald [M2] and (in general) Koornwinder [K2]. The proof for the Wilson 
and continuous Hahn case is very similar to that of the Askey-Wilson case and has been outlined in [D3].

3.1. Askey-Wilson type. The second order $(q$-) difference operator diagonalized by the polynomials $p_{\lambda}^{A W}, \lambda \in \Lambda$, is given by

$$
D^{A W}=\sum_{1 \leq j \leq n}\left(V_{j}^{A W}(x)\left(T_{j, q}-1\right)+V_{-j}^{A W}(x)\left(T_{j, q}^{-1}-1\right)\right)
$$

with

$$
\begin{aligned}
V_{ \pm j}^{A W}(x)= & \frac{\prod_{0 \leq r \leq 3}\left(1-t_{r} e^{ \pm i \alpha x_{j}}\right)}{\left(1-e^{ \pm 2 i \alpha x_{j}}\right)\left(1-q e^{ \pm 2 i \alpha x_{j}}\right)} \\
& \times \prod_{1 \leq k \leq n, k \neq j}\left(\frac{1-t e^{i \alpha\left( \pm x_{j}+x_{k}\right)}}{1-e^{i \alpha\left( \pm x_{j}+x_{k}\right)}}\right)\left(\frac{1-t e^{i \alpha\left( \pm x_{j}-x_{k}\right)}}{1-e^{i \alpha\left( \pm x_{j}-x_{k}\right)}}\right) .
\end{aligned}
$$

Here the operators $T_{j, q}$ act on trigonometric polynomials by means of a $q$-shift of the $j$ th variable,

$$
\left(T_{j, q} f\right)\left(e^{i \alpha x_{1}}, \ldots, e^{i \alpha x_{n}}\right)=f\left(e^{i \alpha x_{1}}, \ldots, e^{i \alpha x_{j-1}}, q e^{i \alpha x_{j}}, e^{i \alpha x_{j+1}}, \ldots, e^{i \alpha x_{n}}\right) .
$$

The eigenvalue of $D^{A W}$ on $p_{\lambda}^{A W}$ has the value

$$
E_{\lambda}^{A W}=\sum_{1 \leq j \leq n}\left(t_{0} t_{1} t_{2} t_{3} q^{-1} t^{2 n-j-1}\left(q^{\lambda_{j}}-1\right)+t^{j-1}\left(q^{-\lambda_{j}}-1\right)\right) .
$$

Proposition 3.1 ([K2]). The multivariable Askey-Wilson polynomials $p_{\lambda}^{A W}, \lambda \in \Lambda$ (2.2), satisfy the second order difference equation

$$
D^{A W} p_{\lambda}^{A W}=E_{\lambda}^{A W} p_{\lambda}^{A W} .
$$

For $n=1$, equation (3.8) reduces to the second order difference equation for the one-variable Askey-Wilson polynomials [AW2, KS]

$$
\begin{array}{r}
\frac{\prod_{0 \leq r \leq 3}\left(1-t_{r} e^{i \alpha x}\right)}{\left(1-e^{2 i \alpha x}\right)\left(1-q e^{2 i \alpha x}\right)}\left(p_{l}^{A W}\left(x+\frac{1}{i \alpha} \log q\right)-p_{l}^{A W}(x)\right) \\
+\frac{\prod_{0 \leq r \leq 3}\left(1-t_{r} e^{-i \alpha x}\right)}{\left(1-e^{-2 i \alpha x}\right)\left(1-q e^{-2 i \alpha x}\right)}\left(p_{l}^{A W}\left(x-\frac{1}{i \alpha} \log q\right)-p_{l}^{A W}(x)\right) \\
=\left(t_{0} t_{1} t_{2} t_{3} q^{-1}\left(q^{l}-1\right)+\left(q^{-l}-1\right)\right) p_{l}^{A W}(x) .
\end{array}
$$

3.2. Wilson type. In the case of Wilson type polynomials the difference operator takes the form

$$
D^{W}=\sum_{1 \leq j \leq n}\left(V_{j}^{W}(x)\left(T_{j}-1\right)+V_{-j}^{W}(x)\left(T_{j}^{-1}-1\right)\right),
$$

where

$$
\begin{aligned}
V_{ \pm j}^{W}(x)= & \frac{\prod_{0 \leq r \leq 3}\left(i \nu_{r} \pm x_{j}\right)}{\left( \pm 2 i x_{j}\right)\left( \pm 2 i x_{j}-1\right)} \\
& \times \prod_{1 \leq k \leq n, k \neq j}\left(\frac{i \nu \pm x_{j}+x_{k}}{ \pm x_{j}+x_{k}}\right)\left(\frac{i \nu \pm x_{j}-x_{k}}{ \pm x_{j}-x_{k}}\right)
\end{aligned}
$$

and the action of $T_{j}$ is given by a unit shift of the $j$ th variable along the imaginary axis,

$$
\left(T_{j} f\right)\left(x_{1}, \ldots, x_{n}\right)=f\left(x_{1}, \ldots, x_{j-1}, x_{j}+i, x_{j+1}, \ldots, x_{n}\right) .
$$


The corresponding eigenvalues now read

$$
E_{\lambda}^{W}=\sum_{1 \leq j \leq n} \lambda_{j}\left(\lambda_{j}+\nu_{0}+\nu_{1}+\nu_{2}+\nu_{3}-1+2(n-j) \nu\right) .
$$

Proposition 3.2 ([D3]). The multivariable Wilson polynomials $p_{\lambda}^{W}, \lambda \in \Lambda(2.2)$, satisfy the second order difference equation

$$
D^{W} p_{\lambda}^{W}=E_{\lambda}^{W} p_{\lambda}^{W}
$$

For $n=1$, equation (3.14) reduces to the second order difference equation for the one-variable Wilson polynomials $[\mathrm{KS}]$ :

$$
\begin{gathered}
\frac{\prod_{0 \leq r \leq 3}\left(i \nu_{r}+x\right)}{2 i x(2 i x-1)}\left(p_{l}^{W}(x+i)-p_{l}^{W}(x)\right) \\
+\frac{\prod_{0 \leq r \leq 3}\left(i \nu_{r}-x\right)}{2 i x(2 i x+1)}\left(p_{l}^{W}(x-i)-p_{l}^{W}(x)\right) \\
=l\left(l+\nu_{0}+\nu_{1}+\nu_{2}+\nu_{3}-1\right) p_{l}^{W}(x) .
\end{gathered}
$$

3.3. Continuous Hahn type. For the continuous Hahn type one has

$$
D^{c H}=\sum_{1 \leq j \leq n}\left(V_{j,-}^{c H}(x)\left(T_{j}-1\right)+V_{j,+}^{c H}(x)\left(T_{j}^{-1}-1\right)\right)
$$

with

$$
\begin{aligned}
& V_{j,+}^{c H}(x)=\left(\nu_{0}^{+}+i x_{j}\right)\left(\nu_{1}^{+}+i x_{j}\right) \prod_{1 \leq k \leq n, k \neq j}\left(1+\frac{\nu}{i\left(x_{j}-x_{k}\right)}\right), \\
& V_{j,-}^{c H}(x)=\left(\nu_{0}^{-}-i x_{j}\right)\left(\nu_{1}^{-}-i x_{j}\right) \prod_{1 \leq k \leq n, k \neq j}\left(1-\frac{\nu}{i\left(x_{j}-x_{k}\right)}\right) .
\end{aligned}
$$

The action of $T_{j}$ is the same as in the Wilson case (cf. (3.12)), and the eigenvalues are given by

$$
E_{\lambda}^{c H}=\sum_{1 \leq j \leq n} \lambda_{j}\left(\lambda_{j}+\nu_{0}^{+}+\nu_{1}^{+}+\nu_{0}^{-}+\nu_{1}^{-}-1+2(n-j) \nu\right) .
$$

Proposition 3.3 ([D3]). The multivariable continuous Hahn polynomials $p_{\lambda}^{c H}, \lambda \in$ $\Lambda$ (2.2), satisfy the second order difference equation

$$
D^{c H} p_{\lambda}^{c H}=E_{\lambda}^{c H} p_{\lambda}^{c H}
$$

For $n=1$, Equation (3.20) reduces to the second order difference equation for the one-variable continuous Hahn polynomials $[\mathrm{KS}]$

$$
\begin{array}{r}
\left(\nu_{0}^{-}-i x\right)\left(\nu_{1}^{-}-i x\right)\left(p_{l}^{c H}(x+i)-p_{l}^{c H}(x)\right) \\
+\left(\nu_{0}^{+}+i x\right)\left(\nu_{1}^{+}+i x\right)\left(p_{l}^{c H}(x-i)-p_{l}^{c H}(x)\right) \\
=l\left(l+\nu_{0}^{+}+\nu_{1}^{+}+\nu_{0}^{-}+\nu_{1}^{-}-1\right) p_{l}^{c H}(x) .
\end{array}
$$


3.4. Jacobi type. In the case of multivariable Jacobi type polynomials the operator $D$ diagonalized by $p_{\lambda}$ is given by a second order differential operator of the form

$$
\begin{aligned}
D^{J}= & -\sum_{1 \leq j \leq n} \partial_{j}^{2}-\alpha \sum_{1 \leq j \leq n}\left(\nu_{0} \cot \left(\frac{\alpha x_{j}}{2}\right)-\nu_{1} \tan \left(\frac{\alpha x_{j}}{2}\right)\right) \partial_{j} \\
& -\alpha \nu \sum_{1 \leq j<k \leq n}\left(\cot \left(\frac{\alpha}{2}\left(x_{j}+x_{k}\right)\right)\left(\partial_{j}+\partial_{k}\right)+\cot \left(\frac{\alpha}{2}\left(x_{j}-x_{k}\right)\right)\left(\partial_{j}-\partial_{k}\right)\right),
\end{aligned}
$$

where $\partial_{j} \equiv \partial / \partial x_{j}$. The eigenvalue of $D^{J}$ on $p_{\lambda}^{J}$ takes the value

$$
E_{\lambda}^{J}=\sum_{1 \leq j \leq n} \lambda_{j}\left(\lambda_{j}+\nu_{0}+\nu_{1}+2(n-j) \nu\right) .
$$

Proposition 3.4 ([V, De, H]). The multivariable Jacobi polynomials $p_{\lambda}^{J}, \lambda \in \Lambda$ (2.2), satisfy the second order differential equation

$$
D^{J} p_{\lambda}^{J}=E_{\lambda}^{J} p_{\lambda}^{J} .
$$

For $n=1$, equation (3.24) reduces to the second order differential equation for the one-variable Jacobi polynomials [AbSt, KS]:

$$
\begin{aligned}
-\frac{d^{2} p_{\lambda}^{J}}{d x^{2}}(x) & -\alpha\left(\nu_{0} \cot \left(\frac{\alpha x}{2}\right)-\nu_{1} \tan \left(\frac{\alpha x}{2}\right)\right) \frac{d p_{\lambda}^{J}}{d x}(x) \\
& =l\left(l+\nu_{0}+\nu_{1}\right) p_{l}^{J}(x) .
\end{aligned}
$$

\section{Limit TRANSITIONS}

The operator $D$ of the previous section can be used to arrive at the following useful representation for the polynomials $p_{\lambda}$ (cf. [M2, SK, D5]):

$$
p_{\lambda}=\left(\prod_{\mu \in \Lambda, \mu<\lambda} \frac{D-E_{\mu}}{E_{\lambda}-E_{\mu}}\right) m_{\lambda} .
$$

Indeed, it is not difficult to infer that the r.h.s. of (4.1) determines a polynomial satisfying the defining properties $i$. and $i i$. stated in Section 2. To this end one uses the triangularity (3.2) and symmetry (3.3) of $D$. (It is immediate from the triangularity of $D$ that the r.h.s. of (4.1) can be written as a linear combination of monomials $m_{\mu}$ with $\mu \leq \lambda$; that the r.h.s. is also orthogonal to all $m_{\mu}$ with $\mu<\lambda$ follows from the symmetry of $D$ and the fact that the operator in the numeratorviz. $\prod_{\mu \in \Lambda, \mu<\lambda}\left(D-E_{\mu}\right)$-annihilates the subspace $\operatorname{Span}\left\{m_{\mu}\right\}_{\mu \in \Lambda, \mu<\lambda}$ in view of the Cayley-Hamilton theorem.) Notice to this end also that (generically) the denominators in (4.1) are well-defined because in each of the concrete cases discussed above one has that for generic parameters the eigenvalues of $D$ are nondegenerate: $E_{\mu} \neq E_{\lambda}$ if $\mu \neq \lambda$. The upshot is that the polynomials $p_{\lambda}$ (4.1) coincide with the polynomials defined in Section 2, first for generic parameter values in the domains indicated in Section 2, and then for general parameters in the domain using a continuity argument. (It is immediate from their definition that the polynomials of Section 2 are continuous in the parameters.) In fact, one may employ (4.1) to extend the definition of the multivariable Askey-Wilson, Wilson, continuous Hahn and Jacobi polynomials to generic complex parameter values (cf. [M2, SK, D5], and see also Remark ii. at the end of this section). The results of the current section 
and also those of Sections 5 and 6 then remain true for such extended parameter domains (notice, however, the note at the beginning of Section 6).

Below we will use formula (4.1) to derive limit transitions from the Askey-Wilson type to the Wilson, continuous Hahn and Jacobi type families, respectively. The transition 'Askey-Wilson $\rightarrow$ Jacobi' has already been considered in [M2, D1, SK] and is included here mainly for the sake of completeness. It will be put to use in Section 6 when deriving a system of recurrence relations for the multivariable Jacobi type polynomials.

4.1. Askey-Wilson $\rightarrow$ Wilson. When studying the limit $p_{\lambda}^{A W} \rightarrow p_{\lambda}^{W}$ it is convenient to first express the multivariable Askey-Wilson polynomials in terms of a slightly modified monomial basis consisting of the functions

$$
\tilde{m}_{\lambda}^{A W}(x)=(2 / \alpha)^{2|\lambda|} m_{s y m, \lambda}\left(\sin ^{2}\left(\alpha x_{1} / 2\right), \ldots, \sin ^{2}\left(\alpha x_{n} / 2\right)\right), \quad \lambda \in \Lambda,
$$

where $|\lambda| \equiv \lambda_{1}+\cdots+\lambda_{n}$. Notice that

$$
\lim _{\alpha \rightarrow 0} \tilde{m}_{\lambda}^{A W}(x)=m_{\lambda}^{W}(x),
$$

whereas the original monomials $m_{\lambda}^{A W}(x)(2.4)$ all reduce to constant functions in this limit. Using the relation $\sin ^{2}\left(\alpha x_{j} / 2\right)=1 / 2-\left(e^{i \alpha x_{j}}+e^{-i \alpha x_{j}}\right) / 4$, one easily infers that the bases $\left\{\tilde{m}_{\lambda}^{A W}\right\}_{\lambda \in \Lambda}$ and $\left\{m_{\lambda}^{A W}\right\}_{\lambda \in \Lambda}$ are related by a triangular transformation of the form

$$
\tilde{m}_{\lambda}^{A W}=\left(-1 / \alpha^{2}\right)^{|\lambda|} m_{\lambda}^{A W}+\sum_{\mu \in \Lambda, \mu<\lambda} a_{\lambda, \mu} m_{\mu}^{A W} \quad \text { with } a_{\lambda, \mu} \in \mathbb{R} .
$$

It is clear that in formula (4.1) we may always replace the monomial basis $\left\{m_{\lambda}\right\}_{\lambda \in \Lambda}$ by a different basis that is related by a unitriangular transformation, since (cf. above) the operator $\prod_{\mu \in \Lambda, \mu<\lambda}\left(D-E_{\mu}\right)$ in the numerator of the r.h.s. annihilates the subspace $\operatorname{Span}\left\{m_{\mu}\right\}_{\mu \in \Lambda, \mu<\lambda}$ because of the Cayley-Hamilton theorem. Hence, by taking into account the diagonal matrix elements of the basis transformation (4.4), one sees that formula (4.1) can rewritten in terms of $\tilde{m}_{\lambda}^{A W}$ as

$$
p_{\lambda}^{A W}=\left(-\alpha^{2}\right)^{|\lambda|}\left(\prod_{\mu \in \Lambda, \mu<\lambda} \frac{D^{A W}-E_{\mu}^{A W}}{E_{\lambda}^{A W}-E_{\mu}^{A W}}\right) \tilde{m}_{\lambda}^{A W} .
$$

If we now substitute

$$
q=e^{-\alpha}, \quad t=e^{-\alpha \nu}, \quad t_{r}=e^{-\alpha \nu_{r}} \quad(r=0,1,2,3)
$$

in $D^{A W}$ (3.4) and $E_{\lambda}^{W}(3.7)$, then we have that

$$
\lim _{\alpha \rightarrow 0} \alpha^{-2} D^{A W}=D^{W}, \quad \lim _{\alpha \rightarrow 0} \alpha^{-2} E_{\lambda}^{A W}=E_{\lambda}^{W} .
$$

(Notice to this end that for $q=e^{-\alpha}$ the action of $T_{j, q}$ (3.6) on trigonometric polynomials is the same as that of $T_{j}(3.12)$, i.e., the action amounts to a shift of the variable $x_{j}$ over an imaginary unit: $x_{j} \rightarrow x_{j}+i$. To infer then that in the limit $\alpha \rightarrow 0$ the difference operator $\alpha^{-2} D^{A W}$ formally goes to $D^{W}$ boils down to checking that the coefficients of the operator converge as advertised.)

By applying the limits (4.3) and (4.7) to formula (4.5) we end up with the following limiting relation between the multivariable Askey-Wilson and Wilson type polynomials. 
Proposition 4.1. For Askey-Wilson parameters given by (4.6) one has

$$
p_{\lambda}^{W}(x)=\lim _{\alpha \rightarrow 0}\left(-1 / \alpha^{2}\right)^{|\lambda|} p_{\lambda}^{A W}(x), \quad \lambda \in \Lambda
$$

(with $\left.|\lambda| \equiv \lambda_{1}+\cdots+\lambda_{n}\right)$.

4.2. Askey-Wilson $\rightarrow$ continuous Hahn. Just like in the previous subsection, the derivation of the transition $p_{\lambda}^{A W} \rightarrow p_{\lambda}^{c H}$ hinges again on formula (4.1). If we shift the variables $x_{1}, \ldots, x_{n}$ over a half period by setting

$$
x_{j} \rightarrow x_{j}-\pi /(2 \alpha), \quad j=1, \ldots, n,
$$

and substitute parameters in the following way:

$$
\begin{array}{ll}
q=e^{-\alpha}, & t=e^{-\alpha \nu}, \\
t_{0}=-i e^{-\alpha \nu_{0}^{+}}, & t_{1}=-i e^{-\alpha \nu_{1}^{+}}, \quad t_{2}=i e^{-\alpha \nu_{0}^{-}}, \quad t_{3}=i e^{-\alpha \nu_{1}^{-}},
\end{array}
$$

then the version of formula (4.1) for the multivariable Askey-Wilson polynomials takes the form $\left(e_{j}\right.$ denotes the $j$ th unit vector in the standard basis of $\left.\mathbb{R}^{n}\right)$

$$
p_{\lambda}^{A W}\left(x-\frac{\pi}{2 \alpha}\left(e_{1}+\cdots+e_{n}\right)\right)=\prod_{\mu \in \Lambda, \mu<\lambda}\left(\frac{\tilde{D}^{A W}-E_{\mu}^{A W}}{E_{\lambda}^{A W}-E_{\mu}^{A W}}\right) \tilde{m}_{\lambda}^{A W}(x),
$$

where

$$
\tilde{D}^{A W}=\sum_{1 \leq j \leq n}\left(\tilde{V}_{j}^{A W}(x)\left(T_{j}-1\right)+\tilde{V}_{-j}^{A W}(x)\left(T_{j}^{-1}-1\right)\right)
$$

with

$$
\begin{aligned}
& \tilde{V}_{j}^{A W}(x) \\
& \quad=\frac{\left(1+e^{-\alpha \nu_{0}^{+}} e^{i \alpha x_{j}}\right)\left(1+e^{-\alpha \nu_{1}^{+}} e^{i \alpha x_{j}}\right)\left(1-e^{-\alpha \nu_{0}^{-}} e^{i \alpha x_{j}}\right)\left(1-e^{-\alpha \nu_{1}^{-}} e^{i \alpha x_{j}}\right)}{\left(1+e^{2 i \alpha x_{j}}\right)\left(1+e^{-\alpha} e^{2 i \alpha x_{j}}\right)} \\
& \quad \times \prod_{1 \leq k \leq n, k \neq j}\left(\frac{1+e^{-\alpha \nu} e^{i \alpha\left(x_{j}+x_{k}\right)}}{1+e^{i \alpha\left(x_{j}+x_{k}\right)}}\right)\left(\frac{1-e^{-\alpha \nu} e^{i \alpha\left(x_{j}-x_{k}\right)}}{1-e^{i \alpha\left(x_{j}-x_{k}\right)}}\right), \\
& \quad \tilde{V}_{-j}^{A W}(x) \\
& \quad \times \quad \prod_{1 \leq k \leq n, k \neq j}\left(\frac{\left.1+e^{-\alpha \nu_{0}^{+}} e^{-i \alpha x_{j}}\right)\left(1-e^{-\alpha \nu_{1}^{+}} e^{-i \alpha x_{j}}\right)\left(1+e^{-\alpha \nu_{0}^{-}} e^{-i \alpha x_{j}}\right)\left(1+e^{-\alpha \nu_{1}^{-}} e^{-i \alpha x_{j}}\right)}{\left(1+e^{-2 i \alpha x_{j}}\right)\left(1+e^{-\alpha} e^{-2 i \alpha x_{j}}\right)}\right)\left(\frac{1-e^{-\alpha \nu} e^{-i \alpha\left(x_{j}-x_{k}\right)}}{1-e^{-i \alpha\left(x_{j}-x_{k}\right)}}\right)
\end{aligned}
$$

and

$$
\tilde{m}_{\lambda}^{A W}(x) \equiv m_{\text {sym }, \lambda}\left(2 \sin \left(\alpha x_{1}\right), \ldots, 2 \sin \left(\alpha x_{n}\right)\right) .
$$

(Just as in the case of the transition Askey-Wilson $\rightarrow$ Wilson we have rewritten the operators $T_{j, q}$ (3.6) for $q=e^{-\alpha}$ as $T_{j}$ (3.12).) After dividing by $(2 \alpha)^{|\lambda|}$ the r.h.s. of (4.11) goes for $\alpha \rightarrow 0$ to the corresponding formula for the continuous Hahn polynomials (i.e. with $\tilde{D}^{A W} \rightarrow D^{c H}, E_{\lambda}^{A W} \rightarrow E_{\lambda}^{c H}$ and $(2 \alpha)^{-|\lambda|} \tilde{m}_{\lambda}^{A W} \rightarrow m_{\lambda}^{c H}$ ). Hence, we now arrive at the following limiting relation between the multivariable Askey-Wilson and continuous Hahn type polynomials. 
Proposition 4.2. For Askey-Wilson parameters given by (4.10) one has

$$
p_{\lambda}^{c H}(x)=\lim _{\alpha \rightarrow 0} \frac{1}{(2 \alpha)^{|\lambda|}} p_{\lambda}^{A W}\left(x-\frac{\pi}{2 \alpha} \omega\right), \quad \lambda \in \Lambda,
$$

where $\omega \equiv e_{1}+\cdots+e_{n}$ (with $e_{j}$ denoting the $j$ th unit vector in the standard basis of $\left.\mathbb{R}^{n}\right)$.

4.3. Askey-Wilson $\rightarrow$ Jacobi. To recover the Jacobi type polynomials we substitute the Askey-Wilson parameters

$$
t=q^{g}, \quad t_{0}=q^{g_{0}}, \quad t_{1}=-q^{g_{1}}, \quad t_{2}=q^{g_{0}^{\prime}+1 / 2}, \quad t_{3}=-q^{g_{1}^{\prime}+1 / 2} .
$$

With these parameters the formula of the form (4.1) for the Askey-Wilson type polynomials reduces in the limit $q \rightarrow 1$ to the corresponding formula for the Jacobi type polynomials (i.e., the difference operator $D^{A W}$ with eigenvalues $E_{\lambda}^{A W}$ gets replaced by the differential operator $D^{J}$ with eigenvalues $E_{\lambda}^{J}$ ). The limit $q \rightarrow 1$ amounts to sending the difference step size to zero. In order to analyze the behavior of the operator $D^{A W}$ for $q \rightarrow 1$ in detail it is convenient to substitute $q=e^{-\alpha \beta}$ (so the action of $T_{j, q}(3.6)$ on trigonometric polynomials amounts to the shift $x_{j} \rightarrow$ $\left.x_{j}+i \beta\right)$ and then write formally $T_{j, q}=\exp \left(i \beta \partial_{j}\right)$. A formal expansion in $\beta$ then shows that $D^{A W} \sim \beta^{2} D^{J}$ and that $E_{\lambda}^{A W} \sim \beta^{2} E_{\lambda}^{J}$ for $\beta \rightarrow 0$ (see [D1]). Here the Jacobi parameters $\nu, \nu_{r}$ are related to the parameters $g, g_{r}^{(\prime)}$ in (4.13) via $\nu=g$, $\nu_{0}=g_{0}+g_{0}^{\prime}$ and $\nu_{1}=g_{1}+g_{1}^{\prime}$. As a consequence we obtain the following limiting relation between the multivariable Askey-Wilson and Jacobi type polynomials.

Proposition 4.3 ([M2, D1, SK]). For Askey-Wilson parameters given by (4.13) one has

$$
p_{\lambda}^{J}(x)=\lim _{q \rightarrow 1} p_{\lambda}^{A W}(x)
$$

with the Jacobi parameters $\nu, \nu_{0}$ and $\nu_{1}$ taking the values $g, g_{0}+g_{0}^{\prime}$ and $g_{1}+g_{1}^{\prime}$, respectively.

Remarks. $i$. In the above derivations of Propositions 4.1, 4.2 and 4.3 we have used the fact that the Askey-Wilson type difference operator converges formally (i.e., without specifying the domains of the operators of interest) to the corresponding operators connected with the Wilson, continuous Hahn and Jacobi type polynomials, respectively. In our case such formal limits get their precise meaning when being applied to formula (4.1).

ii. For all our four families $\mathrm{AW}, \mathrm{W}, \mathrm{cH}$ and $\mathrm{J}$ the dependence of (the coefficients of) the operator $D$ and of the eigenvalues $E_{\lambda}$ is polynomial in the parameters $t, t_{0}, t_{1}, t_{2}, t_{3}(\mathrm{AW}), \nu, \nu_{0}, \nu_{1}, \nu_{2}, \nu_{3}(\mathrm{~W}), \nu, \nu_{0}^{ \pm}, \nu_{1}^{ \pm}(\mathrm{cH})$ and $\nu, \nu_{0}, \nu_{1}(\mathrm{~J})$, respectively. Hence, it is immediate from formula (4.1) that (the coefficients of) the polynomials $p_{\lambda}$ are rational in these parameters. We may thus extend the parameter domains for the polynomials given in Section 2 to generic (complex) values by alternatively characterizing $p_{\lambda}$ as the polynomial of the form $p_{\lambda}=m_{\lambda}+\sum_{\mu \in \lambda, \mu<\lambda} c_{\lambda, \mu} m_{\mu}$ satisfying the eigenvalue equation $D p_{\lambda}=E_{\lambda} p_{\lambda}$. In particular, there is with this extended definition no reason to require for the continuous Hahn family that the parameters $\nu_{r}^{+}$and $\nu_{r}^{-}(2.17)$ are related by complex conjugation (the difference operator $D^{c H}$ (3.16) remains triangular when this condition is not met).

It is then clear that also the limit transitions discussed in this section extend to these larger parameter domains of generic (complex) parameter values. Notice, 
however, that the above limit transitions do respect the parameter conditions of Section 2 in the sense that one stays within the specified parameter regimes when performing limit transitions from the Askey-Wilson to Wilson, continuous Hahn and Jacobi type polynomials with parameters taken from their respective orthogonality domains in Section 2.

iii. In the case of one variable the limit transitions from Askey-Wilson polynomials to Wilson, continuous Hahn and Jacobi polynomials were collected in [KS] (together with many other limits between the various (basic) hypergeometric orthogonal families appearing in the ( $q$-)Askey scheme).

\section{Higher order DifFERENCE or DifFERENTIAL EQUATIONS}

In [D1] it was shown that the second order difference equation for the multivariable Askey-Wilson type polynomials can be extended to a system of difference equations having the structure of eigenvalue equations of the form

$$
D_{r} p_{\lambda}=E_{r, \lambda} p_{\lambda}, \quad r=1, \ldots, n,
$$

for $n$ independent commuting difference operators $D_{1}, \ldots, D_{n}$ of order $2, \ldots, 2 n$, respectively. For $r=1$ one recovers the second order difference equation discussed in Section 3.1. After recalling the explicit expressions for the Askey-Wilson type difference operators $D_{r}^{A W}$ and their eigenvalues $E_{r, \lambda}^{A W}$, we will apply the limit transitions of Section 4 to arrive at similar systems of difference equations for the multivariable Wilson and continuous Hahn type polynomials. In case of the transition 'AskeyWilson $\rightarrow$ Jacobi' the step size is sent to zero and the system of difference equations degenerates to a system of hypergeometric differential equations, thus generalizing the state of affairs for the second order operator in the previous section. This limit from Askey-Wilson type difference equations to Jacobi type differential equations has already been discussed in detail in [D1, Sec. 4], so here we will merely state the results and refrain from presenting a complete treatment of this case.

5.1. Askey-Wilson type. The difference operators diagonalized by the multivariable Askey-Wilson polynomials via (5.1) are given by

$$
D_{r}^{A W}=\sum_{\substack{J \subset\{1, \ldots, n\}, 0 \leq|J| \leq r \\ \varepsilon_{j}= \pm 1, j \in J}} U_{J^{c}, r-|J|}^{A W}(x) V_{\varepsilon J, J^{c}}^{A W}(x) T_{\varepsilon J, q}, \quad r=1, \ldots, n,
$$

with

$$
\begin{gathered}
T_{\varepsilon J, q}=\prod_{j \in J} T_{j, q}^{\varepsilon_{j}}, \\
V_{\varepsilon J, K}^{A W}(x)=\prod_{j \in J} w^{A W}\left(\varepsilon_{j} x_{j}\right) \\
\times \prod_{\substack{j, j^{\prime} \in J \\
j<j^{\prime}}} v^{A W}\left(\varepsilon_{j} x_{j}+\varepsilon_{j^{\prime}} x_{j^{\prime}}\right) v^{A W}\left(\varepsilon_{j} x_{j}+\varepsilon_{j^{\prime}} x_{j^{\prime}}-i \log (q) / \alpha\right) \\
\times \prod_{\substack{j \in J \\
k \in K}} v^{A W}\left(\varepsilon_{j} x_{j}+x_{k}\right) v^{A W}\left(\varepsilon_{j} x_{j}-x_{k}\right),
\end{gathered}
$$




$$
\begin{aligned}
U_{K, p}^{A W}(x)=(-1)^{p} & \sum_{\substack{L \subset K,|L|=p \\
\varepsilon_{l}= \pm 1, l \in L}}\left(\prod_{l \in L} w^{A W}\left(\varepsilon_{l} x_{l}\right)\right. \\
& \times \prod_{\substack{l, l^{\prime} \in L \\
l<l^{\prime}}} v^{A W}\left(\varepsilon_{l} x_{l}+\varepsilon_{l^{\prime}} x_{l^{\prime}}\right) v^{A W}\left(-\varepsilon_{l} x_{l}-\varepsilon_{l^{\prime}} x_{l^{\prime}}+i \log (q) / \alpha\right) \\
& \left.\times \prod_{\substack{l \in L \\
k \in K \backslash L}} v^{A W}\left(\varepsilon_{l} x_{l}+x_{k}\right) v^{A W}\left(\varepsilon_{l} x_{l}-x_{k}\right)\right)
\end{aligned}
$$

and

$$
\begin{aligned}
v^{A W}(z) & =t^{-1 / 2}\left(\frac{1-t e^{i \alpha z}}{1-e^{i \alpha z}}\right) \\
w^{A W}(z) & =\left(t_{0} t_{1} t_{2} t_{3} q^{-1}\right)^{-1 / 2} \frac{\prod_{0 \leq r \leq 3}\left(1-t_{r} e^{i \alpha z}\right)}{\left(1-e^{2 i \alpha z}\right)\left(1-q e^{2 i \alpha z}\right)} .
\end{aligned}
$$

Here the action of the operators $T_{j, q}^{ \pm 1}$ is defined in accordance with (3.6). The summation in (5.2) is over all index sets $J \subset\{1, \ldots, n\}$ with cardinality $|J| \leq r$ and over all configurations of signs $\varepsilon_{j} \in\{+1,-1\}$ with $j \in J$. Furthermore, by convention empty products are taken to be equal to one, and $U_{K, p} \equiv 1$ for $p=0$.

The corresponding eigenvalue of $D_{r}^{A W}$ on $p_{\lambda}^{A W}$ has the value

$$
E_{r, \lambda}^{A W}=E_{r}\left(\tau_{1} q^{\lambda_{1}}+\tau_{1}^{-1} q^{-\lambda_{1}}, \ldots, \tau_{n} q^{\lambda_{n}}+\tau_{n}^{-1} q^{-\lambda_{n}} ; \tau_{r}+\tau_{r}^{-1}, \ldots, \tau_{n}+\tau_{n}^{-1}\right),
$$

where

$$
E_{r}\left(x_{1}, \ldots, x_{n} ; y_{r}, \ldots, y_{n}\right)
$$

and

$$
\tau_{j}=t^{n-j}\left(t_{0} t_{1} t_{2} t_{3} q^{-1}\right)^{1 / 2}, \quad j=1, \ldots, n .
$$

(The second sum in (5.6) is understood to be equal to 1 when $|J|=r$.)

Summarizing, we have the following theorem generalizing Proposition 3.1.

Theorem 5.1 ([D1, D4]). The multivariable Askey-Wilson polynomials $p_{\lambda}^{A W}, \lambda \in$ $\Lambda(2.2)$, satisfy a system of difference equations of the form

$$
D_{r}^{A W} p_{\lambda}^{A W}=E_{r, \lambda}^{A W} p_{\lambda}^{A W}, \quad r=1, \ldots, n .
$$

For $r=1$ the difference equation (5.8) goes over in the second order difference equation (3.8) after multiplication by a constant with value $t^{n-1}\left(t_{0} t_{1} t_{2} t_{3} q^{-1}\right)^{1 / 2}$. More generally, one may multiply the difference equation (5.8) for arbitrary $r$ by the constant factor $t^{r(n-1)-r(r-1) / 2}\left(t_{0} t_{1} t_{2} t_{3} q^{-1}\right)^{r / 2}$ to obtain a difference equation that is polynomial in the parameters $t, t_{0}, \ldots, t_{3}$ and rational in $q$. Such multiplication amounts to omitting the factors $t^{-1 / 2}$ and $\left(t_{0} t_{1} t_{2} t_{3} q^{-1}\right)^{-1 / 2}$ in the definition of $v^{A W}(z)$ (5.3) and $w^{A W}(x)$ (5.4) and to replacing the eigenvalues by

$$
\begin{aligned}
E_{r, \lambda}^{A W} \rightarrow & t^{-r(r-1) / 2} \\
& \times E_{r}\left(\tau_{1}^{+} q^{\lambda_{1}}+\tau_{1}^{-} q^{-\lambda_{1}}, \ldots, \tau_{n}^{+} q^{\lambda_{n}}+\tau_{n}^{-} q^{-\lambda_{n}} ; \tau_{r}^{+}+\tau_{r}^{-}, \ldots, \tau_{n}^{+}+\tau_{n}^{-}\right)
\end{aligned}
$$


with

$$
\begin{aligned}
\tau_{j}^{+} & =t^{n-1}\left(t_{0} t_{1} t_{2} t_{3} q^{-1}\right)^{1 / 2} \tau_{j}=t_{0} t_{1} t_{2} t_{3} q^{-1} t^{2 n-1-j}, \\
\tau_{j}^{-} & =t^{n-1}\left(t_{0} t_{1} t_{2} t_{3} q^{-1}\right)^{1 / 2} \tau_{j}^{-1}=t^{j-1} .
\end{aligned}
$$

5.2. Wilson type. If we substitute Askey-Wilson parameters of the form (4.6) and divide by $\alpha^{2 r}$, then for $\alpha \rightarrow 0$ the operator $D_{r}^{A W}$ goes over into

$$
D_{r}^{W}=\sum_{\substack{J \subset\{1, \ldots, n\}, 0 \leq \leq J \mid \leq r \\ \varepsilon_{j}= \pm 1, j \in J}} U_{J^{c}, r-|J|}^{W}(x) V_{\varepsilon J, J^{c}}^{W}(x) T_{\varepsilon J}, \quad r=1, \ldots, n,
$$

with

$$
\begin{aligned}
T_{\varepsilon J}= & \prod_{j \in J} T_{j}^{\varepsilon_{j}}, \\
V_{\varepsilon J, K}^{W}(x)= & \prod_{j \in J} w^{W}\left(\varepsilon_{j} x_{j}\right) \prod_{\substack{j, j^{\prime} \in J \\
j<j^{\prime}}} v^{W}\left(\varepsilon_{j} x_{j}+\varepsilon_{j^{\prime}} x_{j^{\prime}}\right) v^{W}\left(\varepsilon_{j} x_{j}+\varepsilon_{j^{\prime}} x_{j^{\prime}}+i\right) \\
& \times \prod_{\substack{j \in J \\
k \in K}} v^{W}\left(\varepsilon_{j} x_{j}+x_{k}\right) v^{W}\left(\varepsilon_{j} x_{j}-x_{k}\right), \\
U_{K, p}^{W}(x) \quad & \sum_{\substack{L \subset K,|L|=p \\
\varepsilon= \pm 1, l \in L}}\left(\prod_{l \in L} w^{W}\left(\varepsilon_{l} x_{l}\right) \prod_{\substack{l, l^{\prime} \in L \\
l<l^{\prime}}} v^{W}\left(\varepsilon_{l} x_{l}+\varepsilon_{l^{\prime}} x_{l^{\prime}}\right) v^{W}\left(-\varepsilon_{l} x_{l}-\varepsilon_{l^{\prime}} x_{l^{\prime}}-i\right)\right. \\
& \left.\times \prod_{\substack{l \in L \\
k \in K \backslash L}} v^{W}\left(\varepsilon_{l} x_{l}+x_{k}\right) v^{W}\left(\varepsilon_{l} x_{l}-x_{k}\right)\right)
\end{aligned}
$$

and

$$
v^{W}(z)=\frac{i \nu+z}{z}, \quad w^{W}(z)=\frac{\prod_{0 \leq r \leq 3}\left(i \nu_{r}+z\right)}{2 i z(2 i z-1)} .
$$

Here the action of $T_{j}^{ \pm}$is taken to be in accordance with (3.12). To verify this limit it suffices to recall that for $q=e^{-\alpha}$ the action of $T_{j, q}(3.6)$ amounts to that of $T_{j}$ (3.12) and to observe that for parameters (4.6) one has $\lim _{\alpha \rightarrow 0} v^{A W}(z)=v^{W}(z)$ and $\lim _{\alpha \rightarrow 0} \alpha^{-2} w^{A W}(z)=w^{W}(z)$.

The eigenvalues become in this limit

$$
E_{r, \lambda}^{W}=E_{r}\left(\left(\rho_{1}^{W}+\lambda_{1}\right)^{2}, \ldots,\left(\rho_{n}^{W}+\lambda_{n}\right)^{2} ;\left(\rho_{r}^{W}\right)^{2}, \ldots,\left(\rho_{n}^{W}\right)^{2}\right)
$$

with $E_{r}(\cdots ; \cdots)$ taken from $(5.6)$ and

$$
\rho_{j}^{W}=(n-j) \nu+\left(\nu_{0}+\nu_{1}+\nu_{2}+\nu_{3}-1\right) / 2 .
$$

For the eigenvalues the computation verifying the limit is a bit more complicated than for the difference operators; it hinges on the following lemma.

Lemma 5.2 ([D1, Sec. 4.2]). One has

$$
\begin{gathered}
\lim _{\alpha \rightarrow 0} \alpha^{-2 r} E_{r}\left(e^{\alpha x_{1}}+e^{-\alpha x_{1}}, \ldots, e^{\alpha x_{n}}+e^{-\alpha x_{n}} ; e^{\alpha y_{r}}+e^{-\alpha y_{r}}, \ldots, e^{\alpha y_{n}}+e^{-\alpha y_{n}}\right) \\
=E_{r}\left(x_{1}^{2}, \ldots, x_{n}^{2} ; y_{r}^{2}, \ldots, y_{n}^{2}\right) .
\end{gathered}
$$


We may thus conclude that the transition $\mathrm{AW} \rightarrow \mathrm{W}$ gives rise to the following generalization of Proposition 3.2.

Theorem 5.3. The multivariable Wilson polynomials $p_{\lambda}^{W}, \lambda \in \Lambda$ (2.2), satisfy a system of difference equations of the form

$$
D_{r}^{W} p_{\lambda}^{W}=E_{r, \lambda}^{W} p_{\lambda}^{W}, \quad r=1, \ldots, n .
$$

For $r=1$ the difference equation (5.13) coincides with the second order difference equation (3.14).

5.3. Continuous Hahn type. After shifting over a half period as in (4.9) and choosing Askey-Wilson parameters of the form (4.10), the operators $\alpha^{-2 r} D_{r}^{A W}$ for $\alpha \rightarrow 0$ go over into

$$
D_{r}^{c H}=\sum_{\substack{J_{+}, J_{-} \subset\{1, \ldots, n\} \\ J_{+} \cap J_{-}=\emptyset,\left|J_{+}\right|+\left|J_{-}\right| \leq r}} U_{J_{+}^{c} \cap J_{-}^{c}, r-\left|J_{+}\right|-\left|J_{-}\right|}^{c H}(x) V_{J_{+}, J_{-} ; J_{+}^{c} \cap J_{-}^{c}}^{c H}(x) T_{J_{+}, J_{-}},
$$

$r=1, \ldots, n$, where

$$
\begin{gathered}
T_{J_{+}, J_{-}}=\prod_{j \in J_{+}} T_{j}^{-1} \prod_{j \in J_{-}} T_{j}, \\
V_{J_{+}, J_{-} ; K}^{c H}(x)=\prod_{j \in J_{+}} w_{+}^{c H}\left(x_{j}\right) \prod_{j \in J_{-}} w_{-}^{c H}\left(x_{j}\right) \\
\quad \times \prod_{\substack{j \in J_{+}, j^{\prime} \in J_{-} \\
c}} v^{c H}\left(x_{j}-x_{j^{\prime}}\right) v^{c H}\left(x_{j}-x_{j^{\prime}}-i\right) \\
\times \prod_{\substack{j \in J_{+} \\
k \in K}} v^{c H}\left(x_{j}-x_{k}\right) \prod_{\substack{j \in J_{-} \\
k \in K^{\prime}}} v^{c H}\left(x_{k}-x_{j}\right), \\
U_{K, p}^{c H}(x)=(-1)^{p} \sum_{L_{+}, L_{-} \subset K, L_{+} \cap L_{+} \cap L_{-}=\emptyset}\left(\prod_{l \in L_{+}} w_{+}^{c H}\left(x_{l}\right) \prod_{l \in L_{-}} w_{-}^{c H}\left(x_{l}\right)\right. \\
\times \prod_{\substack{l \in L_{+} \\
l^{\prime} \in L_{-}}}^{c H}\left(x_{l}-x_{l^{\prime}}\right) v^{c H}\left(x_{l^{\prime}}-x_{l}+i\right) \\
\left.\times \prod_{\substack{l \in L_{+} \\
k \in K \backslash L_{+} \cup L_{-}}} v^{c H}\left(x_{l}-x_{k}\right) \prod_{\substack{l \in L_{-} \\
k \in K \backslash L_{+} \cup L_{-}}} v^{c H}\left(x_{k}-x_{l}\right)\right),
\end{gathered}
$$

and

$$
\begin{gathered}
v^{c H}(z)=\left(1+\frac{\nu}{i z}\right), \\
w_{+}^{c H}(z)=\left(\nu_{0}^{+}+i z\right)\left(\nu_{1}^{+}+i z\right), \quad w_{-}^{c H}(z)=\left(\nu_{0}^{-}-i z\right)\left(\nu_{1}^{-}-i z\right) .
\end{gathered}
$$

(The action of the operators $T_{j}^{ \pm}$is again in accordance with (3.12).) To verify this transition one uses that $v^{A W}\left( \pm\left(x_{j}+x_{k}\right)\right) \rightarrow 1, v^{A W}\left(x_{j}-x_{k}\right) \rightarrow v^{c H}\left(x_{k}-x_{j}\right)$ and $\alpha^{-2} w^{A W}\left( \pm x_{j}\right) \rightarrow w_{\mp}^{c H}\left(x_{j}\right)$, if one sends $\alpha$ to zero after having substituted (4.9) and (4.10). 
The computation of the limit of the eigenvalues is exactly the same as in the Wilson case, and the result reads

$$
E_{r, \lambda}^{c H}=E_{r}\left(\left(\rho_{1}^{c H}+\lambda_{1}\right)^{2}, \ldots,\left(\rho_{n}^{c H}+\lambda_{n}\right)^{2} ;\left(\rho_{r}^{c H}\right)^{2}, \ldots,\left(\rho_{n}^{c H}\right)^{2}\right)
$$

with $E_{r}(\cdots ; \cdots)$ taken from $(5.6)$ and

$$
\rho_{j}^{c H}=(n-j) \nu+\left(\nu_{0}^{+}+\nu_{1}^{+}+\nu_{0}^{-}+\nu_{1}^{-}-1\right) / 2 .
$$

Hence, we arrive the following generalization of Proposition 3.3.

Theorem 5.4. The multivariable continuous Hahn polynomials $p_{\lambda}^{c H}, \lambda \in \Lambda(2.2)$, satisfy a system of difference equations of the form

$$
D_{r}^{c H} p_{\lambda}^{c H}=E_{r, \lambda}^{c H} p_{\lambda}^{c H}, \quad r=1, \ldots, n .
$$

For $r=1$ the difference equation (5.19) coincides with the second order difference equation (3.20).

5.4. Jacobi type. After substituting Askey-Wilson parameters given by (4.13) and dividing by a constant with value $(1-q)^{2 r}$, the $r$ th difference equation in Theorem 5.1 for the Askey-Wilson type polynomials goes for $q \rightarrow 1$ over into a differential equation $D_{r}^{J} p_{\lambda}^{J}=E_{r, \lambda}^{J} p_{\lambda}^{J}$ of order $2 r$ for the multivariable Jacobi type polynomials [D1, Sec. 4]. The computation of the eigenvalue $E_{r, \lambda}^{J}=$ $\lim _{q \rightarrow 1}(1-q)^{-2 r} E_{r, \lambda}^{A W}$ hinges again on Lemma 5.2 , and the result is

$$
E_{r, \lambda}^{J}=E_{r}\left(\left(\rho_{1}^{J}+\lambda_{1}\right)^{2}, \ldots,\left(\rho_{n}^{J}+\lambda_{n}\right)^{2} ;\left(\rho_{r}^{J}\right)^{2}, \ldots,\left(\rho_{n}^{J}\right)^{2}\right)
$$

with $E_{r}(\cdots ; \cdots)$ taken from $(5.6)$ and

$$
\rho_{j}^{J}=(n-j) \nu+\left(\nu_{0}+\nu_{1}\right) / 2,
$$

where $\nu=g, \nu_{0}=g_{0}+g_{0}^{\prime}$ and $\nu_{1}=g_{1}+g_{1}^{\prime}$. For $r=1$ the differential operator $D_{r}^{J}=\lim _{q \rightarrow 1}(1-q)^{-2 r} D_{r}^{A W}$ is given by $D^{J}(3.22)$. More generally, one has that $D_{r}^{J}$ is of the form

$$
D_{r}^{J}=\sum_{\substack{J \subset\{1, \ldots, n\} \\|J|=r}} \prod_{j \in J} \partial_{j}^{2}+\text { l.o. }
$$

(where l.o. stands for the parts of lower order in the partials), but it seems difficult to obtain the relevant differential operators for arbitrary $r$ in explicit form starting from $D_{r}^{A W}(5.2)$.

Theorem 5.5 ([D1, Sec. 4]). The multivariable Jacobi polynomials $p_{\lambda}^{J}, \lambda \in \Lambda(2.2)$, satisfy a system of differential equations of the form

$$
D_{r}^{J} p_{\lambda}^{J}=E_{r, \lambda}^{J} p_{\lambda}^{J}, \quad r=1, \ldots, n,
$$

where $D_{r}^{J}=\lim _{q \rightarrow 1}(1-q)^{-2 r} D_{r}^{A W}$ is of the form (5.22) and the corresponding eigenvalues are given by (5.20).

For $r=1$ the differential equation (5.23) coincides with the second order differential equation (3.24).

Remarks. $i$. The proof in [D1, D4] demonstrating that the multivariable AskeyWilson type polynomials satisfy the system of difference equations in Theorem 5.1 runs along the same lines as the proof for the special case when $r=1$ (Proposition 3.1): it consists of demonstrating that the operator $D_{r}^{A W}$ is triangular with respect to the monomial basis $\left\{m_{\lambda}^{A W}\right\}_{\lambda \in \Lambda}$ and that it is symmetric with respect to 
the inner product $\langle\cdot, \cdot\rangle_{\triangle A W}$. The triangularity proof consists of two parts. First it is shown that $D_{r}^{A W} m_{\lambda}^{A W}$ lies in the space $\mathcal{H}^{A W}$ by inferring that poles originating from the zeros in the denominators of $v^{A W}(z)$ (5.3) and $w^{A W}(z)$ (5.4) all cancel each other. Next, it is verified that the operator $D_{r}^{A W}$ is indeed subtriangular by analyzing the asymptotics for $x$ at infinity.

It is worth noting that if one tries to apply the same approach to arrive at a direct proof of the system of difference equations for the multivariable Wilson and continuous Hahn type polynomials (i.e., without using the fact that these two cases may be seen as limiting cases of the Askey-Wilson type polynomials), then some complications arise. In both cases the proof that the operator $D_{r}$ maps the space $\mathcal{H}$ into itself and that it is symmetric with respect to the inner product $\langle\cdot, \cdot\rangle_{\Delta}$ applies without significant changes. However, it is now not so simple to deduce from the asymptotics at infinity that the difference operator $D_{r}$ is indeed triangular. The reason - in a nutshell - is that the functions $w^{W}(z)(5.10)$ and $w_{ \pm}^{c H}(z)$ (5.16) no longer have constant asymptotics for $z \rightarrow \infty$ (as does $w^{A W}(z)$ for $\left.\exp (i \alpha z) \rightarrow \infty\right)$. As a consequence, it is now much more difficult than for the Askey-Wilson case to rule out the a priori possibility that monomials $m_{\mu}$ with $\mu \not \leq \lambda$ enter the expansion of $D_{r} m_{\lambda}$ in monomial symmetric functions (in principle $D_{r}$ might a priori raise the degrees of the polynomials). For $r=1$ one easily checks by inspection that such $m_{\mu}$ with $\mu \not \leq \lambda$ indeed do not appear in the expansion of $D_{r} m_{\lambda}$, but for general $r$ this is not so easily seen from the explicit expressions at hand.

ii. Some years ago, a rather explicit characterization of a family of commuting differential operators simultaneously diagonalized by the Jacobi type polynomials $p_{\lambda}^{J}$ (and generating the same algebra as the operators $D_{1}^{J}, \ldots, D_{n}^{J}$ ) was presented by Debiard [De]. Alternatively, it also turned out possible to express such differential operators in terms of symmetric functions of Heckman's trigonometric generalization of the Dunkl differential-reflection operators related to the root system $B C_{n}$ $[\mathrm{H}, \mathrm{Du} 2]$. In both cases, however, it is a nontrivial problem to deduce from those results an explicit combinatorial formula for (the coefficients of) the operator $D_{r}^{J}$ for arbitrary $r$. In the case of Debiard's operators one problem is that the corresponding eigenvalues do not seem to be known precisely in closed form (and also that one would like to commute all coefficients to the left); in the case of an expression in terms of trigonometric Dunkl type differential-reflection operators it appears to be difficult to explicitly compute the differential operator corresponding to the restriction of the relevant symmetrized differential-reflection operators to the space of symmetric polynomials (except when the order of the leading symbol is small). In the latter case the problem is that one has to commute all reflection operators to the right (and preferably all coefficients to the left). This poses a combinatorial exercise that seems tractable only for small order of the leading symbol.

iii. If one transforms the operator $D^{J}=D_{1}^{J}$ to a second order differential operator that is self-adjoint in $L^{2}\left(\mathbb{R}^{n}, d x_{1} \cdots d x_{n}\right)$ by conjugating with the square root of the weight function $\Delta^{J}(x)(2.19)$, then one arrives at a Schrödinger operator of the form

$$
\begin{aligned}
& \left(\Delta^{J}\right)^{\frac{1}{2}} D^{J}\left(\Delta^{J}\right)^{-\frac{1}{2}} \\
& =-\sum_{1 \leq j \leq n} \partial_{j}^{2}+\frac{1}{4} \alpha^{2} \sum_{1 \leq j \leq n}\left(\frac{\nu_{0}\left(\nu_{0}-1\right)}{\sin ^{2}\left(\frac{\alpha x_{j}}{2}\right)}+\frac{\nu_{1}\left(\nu_{1}-1\right)}{\cos ^{2}\left(\frac{\alpha x_{j}}{2}\right)}\right) \\
& \quad+\frac{1}{2} \nu(\nu-1) \alpha^{2} \sum_{1 \leq j<k \leq n}\left(\frac{1}{\sin ^{2} \frac{\alpha}{2}\left(x_{j}-x_{k}\right)}+\frac{1}{\sin ^{2} \frac{\alpha}{2}\left(x_{j}-x_{k}\right)}\right)-\varepsilon_{0},
\end{aligned}
$$


where $\varepsilon_{0}=\alpha^{2} \sum_{1 \leq j \leq n}\left(\rho_{j}^{J}\right)^{2}$. Explicit expressions for $n$ independent commuting differential operators generating the same commutative algebra as the transformed operators $\left(\Delta^{J}\right)^{\frac{1}{2}} D_{r}^{J}\left(\Delta^{J}\right)^{-\frac{1}{2}}, r=1, \ldots, n$, can be found in the literature as a special case of the formulas presented in [OOS, OS]. However, just as in the previous remark it is again nontrivial to determine explicitly the relation between our transformed operators $\left(\Delta^{J}\right)^{\frac{1}{2}} D_{r}^{J}\left(\Delta^{J}\right)^{-\frac{1}{2}}, r=1, \ldots, n$, and the relevant specialization of the differential operators in [OOS, OS], because to our knowledge the eigenvalues of the latter operators are not available in closed form (except in cases when the order of the leading symbol is small).

\section{Recurrence Relations}

Note: In this section we assume Askey-Wilson, Wilson and continuous Hahn parameters subject to the contraints $q t_{0} t_{1}^{-1} t_{2}^{-1} t_{3}^{-1}=1,1+\nu_{0}-\nu_{1}-\nu_{2}-\nu_{3}=0$ and $1+\nu_{0}^{+}-\nu_{0}^{-}-\nu_{1}^{+}-\nu_{1}^{-}=0$, respectively. It is possible to extend the results of this section to general parameters by invoking a recent result due to Macdonald (see Remark $i v$, below).

We first recall the system of recurrence relations for the multivariable AskeyWilson type polynomials presented in [D5]. Next, the limit transitions of Section 4 are applied to arrive at similar systems of recurrence relations for the multivariable Wilson, continuous Hahn and Jacobi type polynomials, respectively. To describe these recurrence relations it is convenient to pass from the monic polynomials $p_{\lambda}(x)$ to a different normalization by introducing

$$
P_{\lambda}(x) \equiv c_{\lambda} p_{\lambda}(x), \quad c_{\lambda}=c^{|\lambda|} \frac{\hat{\Delta}_{+}(\rho)}{\hat{\Delta}_{+}(\rho+\lambda)}
$$

where $c$ denotes some constant not depending on $\lambda$ (recall also that $|\lambda| \equiv \lambda_{1}+\cdots+$ $\left.\lambda_{n}\right)$ and the function $\hat{\Delta}_{+}(x)$ is of the form

$$
\hat{\Delta}_{+}(x)=\prod_{1 \leq j<k \leq n} \hat{d}_{v,+}\left(x_{j}+x_{k}\right) \hat{d}_{v,+}\left(x_{j}-x_{k}\right) \prod_{1 \leq j \leq n} \hat{d}_{w,+}\left(x_{j}\right)
$$

The precise value of the constant $c$, the vector $\rho=\left(\rho_{1}, \ldots, \rho_{n}\right)$ and the form of the functions $\hat{d}_{v,+}, \hat{d}_{w,+}$ depends on the type of polynomials of interest and will be detailed below separately for each case.

The general structure of the recurrence relations for the renormalized polynomials $P_{\lambda}(x)$ reads

$$
\hat{E}_{r}(x) P_{\lambda}(x)=\sum_{\substack{J \subset\{1, \ldots, n\}, 0 \leq|J| \leq r \\ \varepsilon_{j}= \pm 1, j \in J ; e_{\varepsilon J}+\lambda \in \Lambda}} \hat{U}_{J^{c}, r-|J|}(\rho+\lambda) \hat{V}_{\varepsilon J, J^{c}}(\rho+\lambda) P_{\lambda+e_{\varepsilon} J}(x)
$$

with $r=1, \ldots, n$, and

$$
e_{\varepsilon J}=\sum_{j \in J} \varepsilon_{j} e_{j}
$$




$$
\begin{aligned}
& \hat{V}_{\varepsilon J, K}(x)= \prod_{j \in J} \hat{w}\left(\varepsilon_{j} x_{j}\right) \prod_{\substack{j, j^{\prime} \in J \\
j<j^{\prime}}} \hat{v}\left(\varepsilon_{j} x_{j}+\varepsilon_{j^{\prime}} x_{j^{\prime}}\right) \hat{v}\left(\varepsilon_{j} x_{j}+\varepsilon_{j^{\prime}} x_{j^{\prime}}+1\right) \\
& \times \prod_{\substack{j \in J \\
k \in K}} \hat{v}\left(\varepsilon_{j} x_{j}+x_{k}\right) \hat{v}\left(\varepsilon_{j} x_{j}-x_{k}\right), \\
& \hat{U}_{K, p}(x) \\
&=(-1)^{p} \sum_{\substack{L \subset K,|L|=p \\
\varepsilon_{l}= \pm 1, l \in L}}\left(\prod_{l \in L} \hat{w}\left(\varepsilon_{l} x_{l}\right) \prod_{\substack{l, l^{\prime} \in L \\
l<l^{\prime}}} \hat{v}\left(\varepsilon_{l} x_{l}+\varepsilon_{l^{\prime}} x_{l^{\prime}}\right) \hat{v}\left(-\varepsilon_{l} x_{l}-\varepsilon_{l^{\prime}} x_{l^{\prime}}-1\right)\right. \\
& \\
&\left.\quad \times \prod_{\substack{l \in L \\
k \in K \backslash L}} \hat{v}\left(\varepsilon_{l} x_{l}+x_{k}\right) \hat{v}\left(\varepsilon_{l} x_{l}-x_{k}\right)\right) .
\end{aligned}
$$

The functions $\hat{E}_{1}(x), \ldots, \hat{E}_{n}(x)$ appearing in the l.h.s. of (6.3) denote certain (explicitly given) symmetric polynomials that generate the algebra of all symmetric polynomials. The $r$ th recurrence relation expresses the fact that the expansion of the product $\hat{E}_{r}(x) P_{\lambda}(x)$ in terms of the basis elements $P_{\mu}(x), \mu \in \Lambda$, is known explicitly (i.e., the coefficients in the expansion are known in closed form). The expansion coefficients are determined by the functions $\hat{v}$ and $\hat{w}$ (together with the vector $\rho$ ) whose precise forms again depend on the class of polynomials of interest. In combinatorics one sometimes refers to this type of recurrence relations as generalized Pieri type formulas, after similar expansion formulas for the products of elementary or complete symmetric functions and Schur functions (in terms of the latter functions) [M4]. In the simplest case, i.e. when $r=1$, the recurrence formula (6.3) reduces to an expression of the form

$$
\begin{aligned}
\hat{E}(x) P_{\lambda}(x)= & \sum_{\substack{1 \leq j \leq n \\
\lambda+e_{j} \in \Lambda}} \hat{V}_{j}(\rho+\lambda)\left(P_{\lambda+e_{j}}(x)-P_{\lambda}(x)\right) \\
& +\sum_{\substack{1 \leq j \leq n \\
\lambda-e_{j} \in \Lambda}} \hat{V}_{-j}(\rho+\lambda)\left(P_{\lambda-e_{j}}(x)-P_{\lambda}(x)\right)
\end{aligned}
$$

with $\hat{E}(x)=\hat{E}_{1}(x)$ and

$$
\hat{V}_{ \pm j}(x)=\hat{w}\left( \pm x_{j}\right) \prod_{1 \leq k \leq n, k \neq j} \hat{v}\left( \pm x_{j}+x_{k}\right) \hat{v}\left( \pm x_{j}-x_{k}\right) .
$$

We will see that for the four families considered below this formula reduces, in the case of one variable, to the well-known three-term recurrence relation for the Askey-Wilson, Wilson, continuous Hahn or Jacobi polynomials, respectively.

6.1. Askey-Wilson type. The normalization constants $c_{\lambda}^{A W}$ for the polynomials in (6.1) of Askey-Wilson type $P_{\lambda}^{A W}(x)$ are determined by the constant $c^{A W}=1$ and the functions

$$
\begin{aligned}
& \hat{d}_{v,+}^{A W}(z)=t^{-z / 2} \frac{\left(q^{z} ; q\right)_{\infty}}{\left(t q^{z} ; q\right)_{\infty}} \\
& \hat{d}_{w,+}^{A W}(z)=\left(\hat{t}_{0} \hat{t}_{1} \hat{t}_{2} \hat{t}_{3} q^{-1}\right)^{-z / 2} \frac{\left(q^{2 z} ; q\right)_{\infty}}{\left(\hat{t}_{0} q^{z}, \hat{t}_{1} q^{z}, \hat{t}_{2} q^{z}, \hat{t}_{3} q^{z} ; q\right)_{\infty}}
\end{aligned}
$$


Here we have introduced dependent parameters $\hat{t}_{r}$ that are related to the AskeyWilson parameters $t_{r}$ by [M3, D5]

$$
\begin{aligned}
& \hat{t}_{0}=\left(t_{0} t_{1} t_{2} t_{3} q^{-1}\right)^{1 / 2} \\
& \hat{t}_{1}=\left(t_{0} t_{1} t_{2}^{-1} t_{3}^{-1} q\right)^{1 / 2} \\
& \hat{t}_{2}=\left(t_{0} t_{1}^{-1} t_{2} t_{3}^{-1} q\right)^{1 / 2} \\
& \hat{t}_{3}=\left(t_{0} t_{1}^{-1} t_{2}^{-1} t_{3} q\right)^{1 / 2}
\end{aligned}
$$

The vector $\rho=\rho^{A W}=\left(\rho_{1}^{A W}, \ldots, \rho_{n}^{A W}\right)$ has in the present case components of the form

$$
\rho_{j}^{A W}={ }^{q} \log \tau_{j}, \quad \tau_{j}=t^{n-j}\left(t_{0} t_{1} t_{2} t_{3} q^{-1}\right)^{1 / 2},
$$

and is introduced mostly for notational convenience. In fact, the logarithm with base $q$ entering through the components $\rho_{j}^{A W}$ merely has a formal meaning and appears in our formulas always as an exponent of $q$.

The symmetric functions $\hat{E}_{r}^{A W}(x)$ multiplying $P_{\lambda}^{A W}(x)$ at the l.h.s. of (6.3) are given by

$$
\hat{E}_{r}^{A W}(x)=E_{r}\left(e^{i \alpha x_{1}}+e^{-i \alpha x_{1}}, \ldots, e^{i \alpha x_{n}}+e^{-i \alpha x_{n}} ; \hat{\tau}_{r}+\hat{\tau}_{r}^{-1}, \ldots, \hat{\tau}_{n}+\hat{\tau}_{n}^{-1}\right)
$$

with $E_{r}(\cdots ; \cdots)$ being taken from (5.6) (as usual) and with

$$
\hat{\tau}_{j}=t^{n-j}\left(\hat{t}_{0} \hat{t}_{1} \hat{t}_{2} \hat{t}_{3} q^{-1}\right)^{1 / 2}
$$

(cf. (6.9)). The coefficients at the r.h.s. of (6.3) entering the expansion of the function $\hat{E}_{r}^{A W}(x) P_{\lambda}^{A W}(x)$ in terms of the basis elements $P_{\mu}^{A W}(x)$ are characterized by the functions

$$
\begin{aligned}
\hat{v}^{A W}(z) & =t^{-1 / 2}\left(\frac{1-t q^{z}}{1-q^{z}}\right) \\
\hat{w}^{A W}(z) & =\left(\hat{t}_{0} \hat{t}_{1} \hat{t}_{2} \hat{t}_{3} q^{-1}\right)^{-1 / 2} \frac{\prod_{0 \leq r \leq 3}\left(1-\hat{t}_{r} q^{z}\right)}{\left(1-q^{2 z}\right)\left(1-q^{2 z+1}\right)}
\end{aligned}
$$

(cf. (5.3), (5.4)). We now have the following theorem from [D5].

Theorem 6.1 ([D5]). The renormalized multivariable Askey-Wilson type polynomials $P_{\lambda}^{A W}(x), \lambda \in \Lambda$ (2.2), satisfy a system of recurrence relations given by (6.3) with $\hat{E}_{r}^{A W}, \hat{v}^{A W}, \hat{w}^{A W}$ and $\rho^{A W}$ taken from (6.10), (6.12), (6.13) and (6.9) (and with $r=1, \ldots, n)$.

For $r=1$ one has

$$
\hat{E}^{A W}(x)=\hat{E}_{1}^{A W}(x)=\sum_{1 \leq j \leq n}\left(e^{i \alpha x_{j}}+e^{-i \alpha x_{j}}-\hat{\tau}_{j}-\hat{\tau}_{j}^{-1}\right) .
$$

The corresponding recurrence formula (6.4), (6.5) specializes in the case of one variable to the three-term recurrence relation for the renormalized Askey-Wilson polynomials

$$
P_{l}^{A W}(x)={ }_{4} \phi_{3}\left(\begin{array}{c}
q^{-l}, t_{0} t_{1} t_{2} t_{3} q^{l-1}, t_{0} e^{i \alpha x}, t_{0} e^{-i \alpha x} \\
t_{0} t_{1}, t_{0} t_{2}, t_{0} t_{3}
\end{array} ; q, q\right)
$$


which reads [AW2, KS]

$$
\begin{aligned}
& \left(2 \cos (\alpha x)-t_{0}-t_{0}^{-1}\right) P_{l}^{A W}(x) \\
& =\frac{\left(1-t_{0} t_{1} t_{2} t_{3} q^{l-1}\right) \prod_{1 \leq r \leq 3}\left(1-t_{0} t_{r} q^{l}\right)}{t_{0}\left(1-t_{0} t_{1} t_{2} t_{3} q^{2 l-1}\right)\left(1-t_{0} t_{1} t_{2} t_{3} q^{2 l}\right)}\left(P_{l+1}^{A W}(x)-P_{l}^{A W}(x)\right) \\
& +\frac{t_{0}\left(1-q^{l}\right) \prod_{1 \leq r<s \leq 3}\left(1-t_{s} t_{r} q^{l-1}\right)}{\left(1-t_{0} t_{1} t_{2} t_{3} q^{2 l-2}\right)\left(1-t_{0} t_{1} t_{2} t_{3} q^{2 l-1}\right)}\left(P_{l-1}^{A W}(x)-P_{l}^{A W}(x)\right) .
\end{aligned}
$$

It is important to observe that, despite the appearances of square roots (and infinite products) in intermediate expressions, both the normalization constants $c_{\lambda}^{A W}$ and the Askey-Wilson type recurrence relations (6.3) are in the end rational in $q$ and the parameters $t, t_{0}, \ldots, t_{3}$. For the recurrence relations this is is rather immediate from the fact that

$$
\begin{aligned}
& \hat{v}^{A W}\left(\varepsilon_{j}\left(\rho_{j}^{A W}+\lambda_{j}\right)+\varepsilon_{k}\left(\rho_{k}^{A W}+\lambda_{k}\right)\right)=t^{-1 / 2}\left(\frac{1-t \tau_{j}^{\varepsilon_{j}} \tau_{k}^{\varepsilon_{k}} q^{\varepsilon_{j} \lambda_{j}+\varepsilon_{K} \lambda_{k}}}{1-\tau_{j}^{\varepsilon_{j}} \tau_{k}^{\varepsilon_{k}} q^{\varepsilon_{j} \lambda_{j}+\varepsilon_{K} \lambda_{k}}}\right) \\
& \hat{w}^{A W}\left(\varepsilon_{j}\left(\rho_{j}^{A W}+\lambda_{j}\right)\right)=\left(\hat{t}_{0} \hat{t}_{1} \hat{t}_{2} \hat{t}_{3} q^{-1}\right)^{-1 / 2} \frac{\prod_{0 \leq r \leq 3}\left(1-\hat{t}_{r} \tau_{j}^{\varepsilon_{j}} q^{\varepsilon_{j} \lambda_{j}}\right)}{\left(1-\tau_{j}^{2 \varepsilon_{j}} q^{2 \varepsilon_{j} \lambda_{j}}\right)\left(1-\tau_{j}^{2 \varepsilon_{j}} q^{2 \varepsilon_{j} \lambda_{j}+1}\right)}
\end{aligned}
$$

The point is that the combinations $\tau_{j}^{\varepsilon_{j}} \tau_{k}^{\varepsilon_{k}}, \hat{t}_{r} \tau_{j}^{\varepsilon_{j}}$ and $\tau_{j}^{2 \varepsilon_{j}}$ are rational in $t, t_{0}, \ldots, t_{3}$, and that the functions $\hat{v}^{A W}$ always emerge in pairs in (6.3) and $\left(\hat{t}_{0} \hat{t}_{1} \hat{t}_{2} \hat{t}_{3} q^{-1}\right)^{1 / 2}=t_{0}$ (hence the square root constant factors in $\hat{v}^{A W}, \hat{w}^{A W}$ do not spoil the rationality). The last equality is also needed to see that $\hat{E}_{r}^{A W}(x)(6.10)$ at the l.h.s. of the recurrence relation depends rationally on the parameters: $\hat{\tau}_{j}=t^{n-j}\left(\hat{t}_{0} \hat{t}_{1} \hat{t}_{2} \hat{t}_{3} q^{-1}\right)^{1 / 2}=$ $t^{n-j} t_{0}$. For $c_{\lambda}^{A W}$ the rationality in the parameters is seen similarly after rewriting in the form (by cancelling common factors in numerator and denominator)

$$
\begin{aligned}
c_{\lambda}^{A W}= & C \prod_{1 \leq j<k \leq n} \frac{\left(\tau_{j} \tau_{k} ; q\right)_{\lambda_{j}+\lambda_{k}}}{\left(t \tau_{j} \tau_{k} ; q\right)_{\lambda_{j}+\lambda_{k}}} \frac{\left(\tau_{j} \tau_{k}^{-1} ; q\right)_{\lambda_{j}-\lambda_{k}}}{\left(t \tau_{j} \tau_{k}^{-1} ; q\right)_{\lambda_{j}-\lambda_{k}}} \\
& \times \prod_{1 \leq j \leq n} \frac{\left(\tau_{j}^{2} ; q\right)_{2 \lambda_{j}}}{\left(\hat{t}_{0} \tau_{j}, \hat{t}_{1} \tau_{j}, \hat{t}_{2} \tau_{j}, \hat{t}_{3} \tau_{j} ; q\right)_{\lambda_{j}}}
\end{aligned}
$$

where $C=\prod_{1 \leq j \leq n} \hat{\tau}_{j}^{\lambda_{j}}$.

6.2. Wilson type. If we substitute Askey-Wilson parameters in accordance with (4.6), then the polynomials $P_{\lambda}^{A W}(x)$ converge for $\alpha \rightarrow 0$ to renormalized multivariable Wilson type polynomials $P_{\lambda}^{W}(x)=c_{\lambda}^{W} p_{\lambda}(x)$. The normalization constant $c_{\lambda}^{W}$ is of the form in (6.1), (6.2) with $c^{W}=-1$, the vector $\rho^{W}$ taken from (5.12), and with the functions $\hat{d}_{v,+}^{W}, \hat{d}_{w,+}^{W}$ given by

$$
\hat{d}_{v,+}^{W}(z)=\frac{\Gamma(\nu+z)}{\Gamma(z)}, \quad \hat{d}_{w,+}^{W}(z)=\frac{\prod_{0 \leq r \leq 3} \Gamma\left(\hat{\nu}_{r}+z\right)}{\Gamma(2 z)} .
$$

Here we have introduced dependent parameters $\hat{\nu}_{r}$ related to the Wilson parameters $\nu_{r}$ by

$$
\begin{aligned}
& \hat{\nu}_{0}=\left(\nu_{0}+\nu_{1}+\nu_{2}+\nu_{3}-1\right) / 2, \\
& \hat{\nu}_{1}=\left(\nu_{0}+\nu_{1}-\nu_{2}-\nu_{3}+1\right) / 2, \\
& \hat{\nu}_{2}=\left(\nu_{0}-\nu_{1}+\nu_{2}-\nu_{3}+1\right) / 2, \\
& \hat{\nu}_{3}=\left(\nu_{0}-\nu_{1}-\nu_{2}+\nu_{3}+1\right) / 2 .
\end{aligned}
$$


To verify that the Askey-Wilson type polynomials $P_{\lambda}^{A W}(x)$ with parameters (4.6) indeed converge for $\alpha \rightarrow 0$ to the renormalized Wilson type polynomials $P_{\lambda}^{W}(x)$ thus defined, one uses Proposition 4.1 and the fact that (for parameters (4.6)) $\lim _{\alpha \rightarrow 0}\left(-\alpha^{2}\right)^{|\lambda|} c_{\lambda}^{A W}=c_{\lambda}^{W}$. The latter limit can be easily checked with the aid of representation (6.18) for $c_{\lambda}^{A W}$, which entails

$$
\begin{aligned}
\lim _{\alpha \rightarrow 0}\left(-\alpha^{2}\right)^{|\lambda|} c_{\lambda}^{A W}= & (-1)^{|\lambda|} \prod_{1 \leq j<k \leq n} \frac{\left(\rho_{j}^{W}+\rho_{k}^{W}\right)_{\lambda_{j}+\lambda_{k}}}{\left(\nu+\rho_{j}^{W}+\rho_{k}^{W}\right)_{\lambda_{j}+\lambda_{k}}} \frac{\left(\rho_{j}^{W}-\rho_{k}^{W}\right)_{\lambda_{j}-\lambda_{k}}}{\left(\nu+\rho_{j}^{W}-\rho_{k}^{W}\right)_{\lambda_{j}-\lambda_{k}}} \\
& \times \prod_{1 \leq j \leq n} \frac{\left(2 \rho_{j}^{W}\right)_{2 \lambda_{j}}}{\left(\hat{\nu}_{0}+\rho_{j}^{W}, \hat{\nu}_{1}+\rho_{j}^{W}, \hat{\nu}_{2}+\rho_{j}^{W}, \hat{\nu}_{3}+\rho_{j}^{W}\right)_{\lambda_{j}}} .
\end{aligned}
$$

It is not difficult to see that the r.h.s. of this expression indeed coincides with the above defined $c_{\lambda}^{W}$ of the form (6.1), (6.2) by rewriting all Pochhammer symbols as a quotient of two gamma functions.

Let us next turn to the limiting behavior of the recurrence relations. For parameters as in (4.6) and after division by $\alpha^{2 r}$ the $r$ th recurrence relation for the Askey-Wilson type polynomial $P_{\lambda}^{A W}(x)$ goes over in a recurrence relation of the form in (6.3) for the Wilson type polynomial $P_{\lambda}^{W}(x)$. The relevant symmetric function at the l.h.s. of the resulting Wilson type recurrence relation is given by

$$
\hat{E}_{r}^{W}(x)=(-1)^{r} \sum_{\substack{J \subset\{1, \ldots, n\} \\ 0 \leq|J| \leq r}} \prod_{j \in J} x_{j}^{2} \sum_{r \leq l_{1} \leq \cdots \leq l_{r-|J|} \leq n}\left(\hat{\rho}_{l_{1}}^{W} \cdots \hat{\rho}_{l_{r-|J|}}^{W}\right)^{2}
$$

with

$$
\hat{\rho}_{j}^{W}=(n-j) \nu+\left(\hat{\nu}_{0}+\hat{\nu}_{1}+\hat{\nu}_{2}+\hat{\nu}_{3}-1\right) / 2
$$

(cf. (5.12)). Furthermore, the coefficients at the r.h.s. of this recurrence relation are determined by the vector $\rho^{W}(5.12)$ and the functions

$$
\hat{v}^{W}(z)=\frac{\nu+z}{z}, \quad \hat{w}^{W}(z)=\frac{\prod_{0 \leq r \leq 3}\left(\hat{\nu}_{r}+z\right)}{2 z(2 z+1)}
$$

(cf. (5.10)). To check this transition from the Askey-Wilson to the Wilson type recurrence relations one uses the fact that for Askey-Wilson parameters given by (4.6) one has

$$
\lim _{\alpha \rightarrow 0} \alpha^{-2 r} \hat{E}_{r}^{A W}(x)=\hat{E}_{r}^{W}(x)
$$

(in view of Lemma 5.2) and that

$$
\begin{aligned}
& \lim _{\alpha \rightarrow 0} \hat{v}^{A W}\left(\varepsilon_{j} \rho_{j}^{A W}+\varepsilon_{k} \rho_{k}^{A W}+z\right)=\hat{v}^{W}\left(\varepsilon_{j} \rho_{j}^{W}+\varepsilon_{k} \rho_{k}^{W}+z\right), \\
& \lim _{\alpha \rightarrow 0} \alpha^{-2} \hat{w}^{A W}\left(\varepsilon_{j} \rho_{j}^{A W}+z\right)=\hat{w}^{W}\left(\varepsilon_{j} \rho_{j}^{W}+z\right) .
\end{aligned}
$$

We have thus derived the following theorem.

Theorem 6.2. The renormalized multivariable Wilson type polynomials $P_{\lambda}^{W}(x)$, $\lambda \in \Lambda$ (2.2), satisfy a system of recurrence relations given by (6.3) with $\hat{E}_{r}^{W}, \hat{v}^{W}$, $\hat{w}^{W}$ and $\rho^{W}$ taken from (6.21), (6.23) and (5.12) (and with $r=1, \ldots, n$ ).

For $r=1$ the recurrence formula is given by (6.4), (6.5) with

$$
\hat{E}^{W}(x)=\hat{E}_{1}^{W}(x)=-\sum_{1 \leq j \leq n}\left(x_{j}^{2}+\left(\hat{\rho}_{j}^{W}\right)^{2}\right) .
$$


In the case of one variable this recurrence formula specializes to the three-term recurrence relation for the renormalized Wilson polynomials:

$$
P_{l}^{W}(x)={ }_{4} F_{3}\left(\begin{array}{c}
-l, \nu_{0}+\nu_{1}+\nu_{2}+\nu_{3}+l-1, \nu_{0}+i x, \nu_{0}-i x \\
\nu_{0}+\nu_{1}, \nu_{0}+\nu_{2}, \nu_{0}+\nu_{3}
\end{array} ;\right),
$$

which reads [AW1, KS]

$$
\text { (6.26) } \begin{aligned}
& -\left(x^{2}+\nu_{0}^{2}\right) P_{l}^{W}(x) \\
= & \frac{\left(l+\nu_{0}+\nu_{1}+\nu_{2}+\nu_{3}-1\right) \prod_{1 \leq r \leq 3}\left(l+\nu_{0}+\nu_{r}\right)}{\left(2 l+\nu_{0}+\nu_{1}+\nu_{2}+\nu_{3}-1\right)\left(2 l+\nu_{0}+\nu_{1}+\nu_{2}+\nu_{3}\right)}\left(P_{l+1}^{W}(x)-P_{l}^{W}(x)\right) \\
+ & \frac{l \prod_{1 \leq r<s \leq 3}\left(l+\nu_{s}+\nu_{r}-1\right)}{\left(2 l+\nu_{0}+\nu_{1}+\nu_{2}+\nu_{3}-2\right)\left(2 l+\nu_{0}+\nu_{1}+\nu_{2}+\nu_{3}-1\right)}\left(P_{l-1}^{W}(x)-P_{l}^{W}(x)\right) .
\end{aligned}
$$

6.3. Continuous Hahn type. If we substitute Askey-Wilson parameters given by (4.10) and shift the variables $x_{j}$ over a half period (cf. (4.9)), then for $\alpha \rightarrow 0$ the Askey-Wilson type polynomials $P_{\lambda}^{A W}(x)$ become renormalized multivariable continuous Hahn type polynomials $P_{\lambda}^{c H}(x)=c_{\lambda}^{c H} p_{\lambda}^{c H}(x)$ of the form in (6.1), (6.2) with $c^{c H}=1 / i$, the vector $\rho^{c H}$ taken from (5.18), and with the functions $\hat{d}_{v,+}^{c H}, \hat{d}_{w,+}^{c H}$ being

$$
\hat{d}_{v,+}^{c H}(z)=\frac{\Gamma(\nu+z)}{\Gamma(z)}, \quad \hat{d}_{w,+}^{c H}(z)=\frac{\prod_{0 \leq r \leq 2} \Gamma\left(\hat{\nu}_{r}+z\right)}{\Gamma(2 z)} .
$$

Here we have again employed the parameters $\hat{\nu}_{0}, \ldots, \hat{\nu}_{3}$ that are now related to the Wilson parameters $\nu_{0}^{ \pm}, \nu_{1}^{ \pm}$by

$$
\begin{aligned}
& \hat{\nu}_{0}=\left(\nu_{0}^{+}+\nu_{1}^{+}+\nu_{0}^{-}+\nu_{1}^{-}-1\right) / 2, \\
& \hat{\nu}_{1}=\left(\nu_{0}^{+}-\nu_{1}^{+}+\nu_{0}^{-}-\nu_{1}^{-}+1\right) / 2, \\
& \hat{\nu}_{2}=\left(\nu_{0}^{+}-\nu_{1}^{+}-\nu_{0}^{-}+\nu_{1}^{-}+1\right) / 2, \\
& \hat{\nu}_{3}=\left(\nu_{0}^{+}+\nu_{1}^{+}-\nu_{0}^{-}-\nu_{1}^{-}+1\right) / 2 .
\end{aligned}
$$

(Notice, however, that $\hat{d}_{w,+}^{c H}$ depends only on $\hat{\nu}_{0}, \hat{\nu}_{1}, \hat{\nu}_{2}$ and not on $\hat{\nu}_{3}$, which is merely introduced for convenience and will be used below (cf. (6.30)).) To verify the transition $P_{\lambda}^{A W}(x) \rightarrow P_{\lambda}^{c H}(x)$ one uses Proposition 4.2 and the fact that (for parameters (4.10)) $\lim _{\alpha \rightarrow 0}(2 \alpha)^{|\lambda|} c_{\lambda}^{A W}=c_{\lambda}^{c H}$. The derivation of the limit $(2 \alpha)^{|\lambda|} c_{\lambda}^{A W} \rightarrow c_{\lambda}^{c H}$ is very similar to that of the Wilson case and hinges again on representation (6.18): first one writes $c_{\lambda}^{A W}(6.18)$ explicitly as a rational expression in the Askey-Wilson parameters $t, t_{0}, \ldots, t_{3}$ by invoking the definitions (6.8) and (6.9); next, substituting the parameters (4.10) and sending $\alpha$ to zero after having multiplied by $(2 \alpha)^{|\lambda|}$ entails an expression for $c_{\lambda}^{c H}$ involving Pochhammer symbols:

$$
\begin{aligned}
c_{\lambda}^{c H}= & (1 / i)^{|\lambda|} \prod_{1 \leq j<k \leq n} \frac{\left(\rho_{j}^{c H}+\rho_{k}^{c H}\right)_{\lambda_{j}+\lambda_{k}}}{\left(\nu+\rho_{j}^{c H}+\rho_{k}^{c H}\right)_{\lambda_{j}+\lambda_{k}}} \frac{\left(\rho_{j}^{c H}-\rho_{k}^{c H}\right)_{\lambda_{j}-\lambda_{k}}}{\left(\nu+\rho_{j}^{c H}-\rho_{k}^{c H}\right)_{\lambda_{j}-\lambda_{k}}} \\
& \times \prod_{1 \leq j \leq n} \frac{\left(2 \rho_{j}^{c H}\right)_{2 \lambda_{j}}}{\left(\hat{\nu}_{0}+\rho_{j}^{c H}, \hat{\nu}_{1}+\rho_{j}^{c H}, \hat{\nu}_{2}+\rho_{j}^{c H}\right)_{\lambda_{j}}},
\end{aligned}
$$

which is seen to be equal to the stated expression of the form (6.1), (6.2) by rewriting all Pochhammer symbols as quotients of gamma functions.

The corresponding recurrence relations for the renormalized multivariable continuous Hahn type polynomials $P_{\lambda}^{c H}(x)$ are of the form given by $(6.3)$ with the 
symmetric functions at the l.h.s. given by

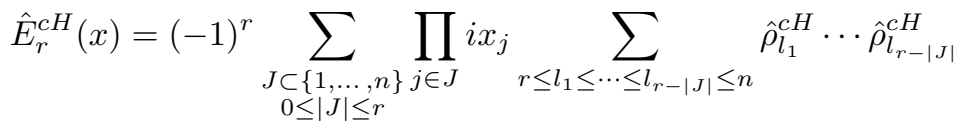

with

$$
\hat{\rho}_{j}^{c H}=(n-j) \nu+\left(\hat{\nu}_{0}+\hat{\nu}_{1}+\hat{\nu}_{2}+\hat{\nu}_{3}-1\right) / 2 .
$$

The coefficients at the r.h.s. are determined by the vector $\rho^{c H}(5.18)$ and the functions

$$
\hat{v}^{c H}(z)=\frac{\nu+z}{z}, \quad \hat{w}^{c H}(z)=\frac{\prod_{0 \leq r \leq 2}\left(\hat{\nu}_{r}+z\right)}{2 z(2 z+1)} .
$$

To arrive at these recurrence relations for the multivariable continuous Hahn type polynomials we have substituted the parameters (4.10), shifted the variables over a half period (4.9) and divided both sides by $(2 i \alpha)^{r}$. For $\alpha \rightarrow 0$ the $r$ th AskeyWilson type recurrence relation then passes over into the $r$ th continuous Hahn type recurrence relation. At this point one uses that (for parameters as in (4.10))

$$
\left.\lim _{\alpha \rightarrow 0}(2 \alpha i)^{-r} \hat{E}_{r}^{A W}\left(x-\frac{\pi}{2 \alpha}\right)\right)=\hat{E}_{r}^{c H}(x)
$$

(which is immediate from the definition of $\hat{E}_{r}^{A W}(x)$ ) and that

$$
\begin{aligned}
& \lim _{\alpha \rightarrow 0} \hat{v}^{A W}\left(\varepsilon_{j} \rho_{j}^{A W}+\varepsilon_{k} \rho_{k}^{A W}+z\right)=\hat{v}^{c H}\left(\varepsilon_{j} \rho_{j}^{c H}+\varepsilon_{k} \rho_{k}^{c H}+z\right), \\
& \lim _{\alpha \rightarrow 0}(2 i \alpha)^{-1} \hat{w}^{A W}\left(\varepsilon_{j} \rho_{j}^{A W}+z\right)=\hat{w}^{c H}\left(\varepsilon_{j} \rho_{j}^{c H}+z\right) .
\end{aligned}
$$

We arrive at the following theorem.

Theorem 6.3. The renormalized multivariable continuous Hahn type polynomials $P_{\lambda}^{c H}(x), \lambda \in \Lambda$ (2.2), satisfy a system of recurrence relations given by (6.3) with $\hat{E}_{r}^{c H}, \hat{v}^{c H}, \hat{w}^{c H}$ and $\rho^{c H}$ taken from (6.29), (6.31) and (5.18) (and with $r=1, \ldots, n)$.

For $r=1$ the recurrence relation is of the form in (6.4), (6.5) with

$$
\hat{E}^{c H}(x)=\hat{E}_{1}^{c H}(x)=-\sum_{1 \leq j \leq n}\left(i x_{j}+\hat{\rho}_{j}^{c H}\right) .
$$

In the case of one variable this formula specializes to the three-term recurrence relation for the renormalized continuous Hahn polynomials:

$$
P_{l}^{c H}(x)={ }_{3} F_{2}\left(\begin{array}{c}
-l, \nu_{0}^{+}+\nu_{0}^{-}+\nu_{1}^{+}+\nu_{1}^{-}+l-1, \nu_{0}^{+}+i x \\
\nu_{0}^{+}+\nu_{0}^{-}, \nu_{0}^{+}+\nu_{1}^{-}
\end{array} ; 1\right),
$$

which reads $[\mathrm{AW} 1, \mathrm{KS}]$

$$
\begin{aligned}
& \quad-\left(i x+\nu_{0}^{+}\right) P_{l}^{c H}(x) \\
& =\frac{\left(l+\nu_{0}^{+}+\nu_{1}^{+}+\nu_{0}^{-}+\nu_{1}^{-}-1\right)\left(l+\nu_{0}^{+}+\nu_{0}^{-}\right)\left(l+\nu_{0}^{+}+\nu_{1}^{-}\right)}{\left(2 l+\nu_{0}^{+}+\nu_{1}^{+}+\nu_{0}^{-}+\nu_{1}^{-}-1\right)\left(2 l+\nu_{0}^{+}+\nu_{1}^{+}+\nu_{0}^{-}+\nu_{1}^{-}\right)} \\
& \quad \times\left(P_{l+1}^{c H}(x)-P_{l}^{c H}(x)\right) \\
& +\frac{-l\left(l+\nu_{1}^{+}+\nu_{0}^{-}-1\right)\left(l+\nu_{1}^{+}+\nu_{1}^{-}-1\right)}{\left(2 l+\nu_{0}^{+}+\nu_{1}^{+}+\nu_{0}^{-}+\nu_{1}^{-}-2\right)\left(2 l+\nu_{0}^{+}+\nu_{1}^{+}+\nu_{0}^{-}+\nu_{1}^{-}-1\right)} \\
& \quad \times\left(P_{l-1}^{c H}(x)-P_{l}^{c H}(x)\right) .
\end{aligned}
$$


6.4. Jacobi type. After substituting the Askey-Wilson parameters (4.13) and sending $q$ to one, the Askey-Wilson type polynomials $P_{\lambda}^{A W}(x)$ pass over to renormalized multivariable Jacobi type polynomials $P_{\lambda}^{J}(x)=c_{\lambda}^{J} p_{\lambda}^{J}(x)$ of the form in (6.1), (6.2) with the Jacobi parameters $\nu_{0}$ and $\nu_{1}$ taking the value $g_{0}+g_{0}^{\prime}$ and $g_{1}+g_{1}^{\prime}$, respectively. The normalization constant $c_{\lambda}^{J}$ is determined by the constant $c^{J}=2^{-2}$ and the vector $\rho^{J}$ taken from (5.21), together with the functions $\hat{d}_{v,+}^{J}$ and $\hat{d}_{w,+}^{J}$ given by

$$
\hat{d}_{v,+}^{J}(z)=\frac{\Gamma(\nu+z)}{\Gamma(z)}, \quad \hat{d}_{w,+}^{J}(z)=\frac{\Gamma\left(\hat{\nu}_{0}+z\right) \Gamma\left(\hat{\nu}_{1}+z\right)}{\Gamma(2 z)} .
$$

Here we have introduced dependent parameters $\hat{\nu}_{0}, \hat{\nu}_{1}$ related to the Jacobi parameters $\nu_{0}, \nu_{1}$ by

$$
\begin{aligned}
& \hat{\nu}_{0}=\left(\nu_{0}+\nu_{1}\right) / 2, \\
& \hat{\nu}_{1}=\left(\nu_{0}-\nu_{1}+1\right) / 2 .
\end{aligned}
$$

The verification of the transition $P_{\lambda}^{A W}(x) \rightarrow P_{\lambda}^{J}(x)$ hinges on Proposition 4.3 and the fact that (for parameters (4.13)) $\lim _{q \rightarrow 1} c_{\lambda}^{A W}=c_{\lambda}^{J}$. The latter limit is again checked using formula (6.18) for $c_{\lambda}^{A W}$, entailing for $q \rightarrow 1$

$$
\begin{aligned}
c_{\lambda}^{J}= & 2^{-2|\lambda|} \prod_{1 \leq j<k \leq n} \frac{\left(\rho_{j}^{J}+\rho_{k}^{J}\right)_{\lambda_{j}+\lambda_{k}}}{\left(\nu+\rho_{j}^{J}+\rho_{k}^{J}\right)_{\lambda_{j}+\lambda_{k}}} \frac{\left(\rho_{j}^{J}-\rho_{k}^{J}\right)_{\lambda_{j}-\lambda_{k}}}{\left(\nu+\rho_{j}^{J}-\rho_{k}^{J}\right)_{\lambda_{j}-\lambda_{k}}} \\
& \times \prod_{1 \leq j \leq n} \frac{\left(2 \rho_{j}^{J}\right)_{2 \lambda_{j}}}{\left(\hat{\nu}_{0}+\rho_{j}^{J}, \hat{\nu}_{1}+\rho_{j}^{J}\right)_{\lambda_{j}}},
\end{aligned}
$$

which is seen to be equal to the stated expression for $c_{\lambda}^{J}$ of the form (6.1), (6.2) by once again rewriting the Pochhammer symbols in terms of gamma functions.

The corresponding recurrence relations for $P_{\lambda}^{J}(x)$ are of the form in (6.3), with the symmetric function at the l.h.s. given by

$$
\hat{E}_{r}^{J}(x)=(-1)^{r} \sum_{\substack{J \subset\{1, \ldots, n\} \\|J|=r}} \prod_{j \in J} \sin ^{2}\left(\frac{\alpha x_{j}}{2}\right)
$$

(this is the $r$ th elementary symmetric function in the variables $-\sin ^{2}\left(\frac{\alpha x_{j}}{2}\right), j=$ $1, \ldots, n)$ and the coefficients at the r.h.s. being determined by $\rho^{J}(5.21)$ and the functions

$$
\hat{v}^{J}(z)=\frac{\nu+z}{z}, \quad \hat{w}^{J}(z)=\frac{\left(\hat{\nu}_{0}+z\right)\left(\hat{\nu}_{1}+z\right)}{2 z(2 z+1)} .
$$

One obtains the recurrence relations for the multivariable Jacobi type polynomials for $q \rightarrow 1$ from the Askey-Wilson type recurrence relations with parameters (4.13) if one divides both sides by $2^{2 r}$. To check this one uses the facts that (for parameters given by (4.13))

$$
\lim _{q \rightarrow 1} 2^{-2 r} \hat{E}_{r}^{A W}(x)=\hat{E}_{r}^{J}(x)
$$

(which is rather immediate from the definition of $\hat{E}_{r}^{A W}(x)$ ) and that

$$
\begin{aligned}
& \lim _{q \rightarrow 1} \hat{v}^{A W}\left(\varepsilon_{j} \rho_{j}^{A W}+\varepsilon_{k} \rho_{k}^{A W}+z\right)=\hat{v}^{J}\left(\varepsilon_{j} \rho_{j}^{J}+\varepsilon_{k} \rho_{k}^{J}+z\right), \\
& \lim _{q \rightarrow 1} 2^{-2} \hat{w}^{A W}\left(\varepsilon_{j} \rho_{j}^{A W}+z\right)=\hat{w}^{J}\left(\varepsilon_{j} \rho_{j}^{J}+z\right) .
\end{aligned}
$$


We thus arrive at the following theorem.

Theorem 6.4. The renormalized multivariable Jacobi type polynomials $P_{\lambda}^{J}(x), \lambda \in$ $\Lambda$ (2.2), satisfy a system of recurrence relations given by (6.3) with $\hat{E}_{r}^{J}, \hat{v}^{J}, \hat{w}^{J}$ and $\rho^{J}$ taken from (6.37), (6.38) and (5.21) (and with $r=1, \ldots, n$ ).

For $r=1$ we now have a recurrence formula of the form in (6.4) with

$$
\hat{E}^{J}(x)=\hat{E}_{1}^{J}(x)=-\sum_{1 \leq j \leq n} \sin ^{2}\left(\frac{\alpha x_{j}}{2}\right) .
$$

In the case of one variable this recurrence formula specializes to the three-term recurrence relation for the renormalized Jacobi polynomials:

$$
P_{l}^{J}(x)={ }_{2} F_{1}\left(\begin{array}{c}
-l, \nu_{0}+\nu_{1}+l \\
\nu_{0}+1 / 2
\end{array} ; \sin ^{2}\left(\frac{\alpha x}{2}\right)\right)
$$

reading $[\mathrm{AbSt}, \mathrm{KS}]$ (notice, however, that our normalization of the polynomials differs slightly from the standard normalization)

$$
\begin{aligned}
& -\sin ^{2}\left(\frac{\alpha x}{2}\right) P_{l}^{J}(x) \\
& =\frac{\left(l+\nu_{0}+\nu_{1}\right)\left(l+\nu_{0}+1 / 2\right)}{\left(2 l+\nu_{0}+\nu_{1}\right)\left(2 l+\nu_{0}+\nu_{1}+1\right)}\left(P_{l+1}^{J}(x)-P_{l}^{J}(x)\right) \\
& +\frac{l\left(l+\nu_{1}-1 / 2\right)}{\left(2 l+\nu_{0}+\nu_{1}\right)\left(2 l+\nu_{0}+\nu_{1}-1\right)}\left(P_{l-1}^{J}(x)-P_{l}^{J}(x)\right) .
\end{aligned}
$$

Remarks. $i$. The combinatorial structure of the recurrence relations for the multivariable Askey-Wilson type polynomials is very similar to that of the difference equations in Section 5. This is by no means a coincidence. In fact, in [D5] the recurrence relations were derived from the difference equations with the aid of a duality property for the renormalized multivariable Askey-Wilson polynomials (which was originally conjectured by Macdonald in [M3] and then proven by Cherednik in [C4] for special parameters corresponding to the reduced root systems (and admissible pairs of the form $\left.\left(R, R^{\vee}\right)\right)$ and next in [D5] for more general parameters subject to the condition in the note at the beginning of this section):

$$
P_{\lambda}^{A W}\left(\frac{i \log (q)}{\alpha}\left(\hat{\rho}^{A W}+\mu\right)\right)=\hat{P}_{\mu}^{A W}\left(\frac{i \log (q)}{\alpha}\left(\rho^{A W}+\lambda\right)\right) .
$$

Here $\hat{P}_{\mu}^{A W}(x), \mu \in \Lambda$, denotes the renormalized multivariable Askey-Wilson type polynomial with the parameters $t_{0}, \ldots, t_{3}$ being replaced by the dual parameters $\hat{t}_{0}, \ldots, \hat{t}_{3}$ (cf. (6.8)), and $\rho^{A W}$ is given by (6.9) whereas $\hat{\rho}^{A W}$ is the corresponding dual vector with $\tau_{j}(6.9)$ replaced by $\hat{\tau}_{j}(6.11)$. If one substitutes

$$
x=\frac{i \log (q)}{\alpha}\left(\rho^{A W}+\lambda\right)
$$

in the $r$ th difference equation of Theorem 5.1 for the polynomial $\hat{P}_{\mu}^{A W}(x)$, then by using property $(6.42 \mathrm{a}$ ) (and the fact that the coefficients have a zero at

$$
x=\frac{i \log (q)}{\alpha}\left(\rho^{A W}+\lambda\right)
$$

if $\left.\lambda+e_{\varepsilon J} \neq \Lambda(2.2)\right)$ one arrives at the $r$ th recurrence relation of Theorem 6.1 (first at the points $x=\frac{i \log (q)}{\alpha}\left(\hat{\rho}^{A W}+\mu\right), \mu \in \Lambda$, and then for arbitrary $x$ using the fact that one deals with an equality between trigonometric polynomials). In the 
case of one variable the duality property in (6.42a) is well-known and immediate (for general parameters) from the explicit expression of the polynomials $P_{\lambda}^{A W}(x)$ in terms of the terminating basic hypergeometric series (6.14).

By applying the transition Askey-Wilson $\rightarrow$ Wilson to $(6.42 \mathrm{a})$ one arrives at a similar duality relation for the multivariable Wilson type polynomials:

$$
P_{\lambda}^{W}\left(i\left(\hat{\rho}^{W}+\mu\right)\right)=\hat{P}_{\mu}^{W}\left(i\left(\rho^{W}+\lambda\right)\right),
$$

where $\hat{P}_{\mu}^{W}(x)$ denotes the Wilson type polynomial with the parameters $\nu_{0}, \ldots, \nu_{3}$ being replaced by $\hat{\nu}_{0}, \ldots, \hat{\nu}_{3}$ (cf. (6.20)). For $n=1$ the duality relation in $(6.42 \mathrm{~b})$ is again well-known and immediate (for general parameters) from the explicit expression of the Wilson polynomials in terms of a terminating hypergeometric series (cf. (6.33)).

ii. For $\mu=0$ the r.h.s. of (6.42a) becomes identical to one, and we arrive at the relation $P_{\lambda}^{A W}\left(i \alpha^{-1} \log \hat{\tau}\right)=1$ (where $\log \hat{\tau}$ stands for the vector $\left(\log \hat{\tau}_{1}, \ldots, \log \hat{\tau}_{n}\right)$ with $\hat{\tau}_{j}$ taken from (6.11)). This equality amounts to the following evaluation (or specialization) formula for the monic Askey-Wilson type polynomials (cf. the definition (6.1))

$$
p_{\lambda}^{A W}\left(i \alpha^{-1} \log \tau\right)=\frac{\hat{\Delta}_{+}^{A W}\left(\rho^{A W}+\lambda\right)}{\hat{\Delta}_{+}^{A W}\left(\rho^{A W}\right)} .
$$

Using the limit transitions of Section 4 one readily arrives at similar evaluation formulas for the multivariable Wilson, continuous Hahn and Jacobi type polynomials:

$$
\begin{aligned}
p_{\lambda}^{W}\left(i \hat{\rho}^{W}\right) & =(-1)^{|\lambda|} \frac{\hat{\Delta}_{+}^{W}\left(\rho^{W}+\lambda\right)}{\hat{\Delta}_{+}^{W}\left(\rho^{W}\right)}, \\
p_{\lambda}^{c H}\left(i \hat{\rho}^{c H}\right) & =i^{|\lambda|} \frac{\hat{\Delta}_{+}^{c H}\left(\rho^{c H}+\lambda\right)}{\hat{\Delta}_{+}^{c H}\left(\rho^{c H}\right)}, \\
p_{\lambda}^{J}(0) & =2^{2|\lambda|} \frac{\hat{\Delta}_{+}^{J}\left(\rho^{J}+\lambda\right)}{\hat{\Delta}_{+}^{J}\left(\rho^{J}\right)} .
\end{aligned}
$$

A proof of this evaluation formula for the Jacobi case by means of shift operators can be found in [Op1]. For the Askey-Wilson case the evaluation formula was conjectured by Macdonald [M2, M3] and then proven by Cherednik [C4] for special parameters related to the reduced root systems (and admissible pairs of the form $\left(R, R^{\vee}\right)$ ) using an extension of Opdam's shift-operator methods. In [D5] a proof of the Askey-Wilson evaluation formula (6.43a) based on the recurrence relations (6.3) was presented (valid for more general parameters subject to the condition in the note at the beginning of the section).

iii. Observe that for Askey-Wilson parameters subject to the condition

$$
q t_{0} t_{1}^{-1} t_{2}^{-1} t_{3}^{-1}=1,
$$

one has (cf. (6.8)) that $\hat{t}_{r}=t_{r}(r=0, \ldots, 3)$ and hence $\hat{P}_{\lambda}^{A W}(x)=P_{\lambda}^{A W}(x)$ (cf. Remark $i$. above). In other words, for these parameters the multivariable AskeyWilson polynomials are self-dual. If we restrict the limit transitions 'Askey-Wilson $\longrightarrow$ Wilson', 'Askey-Wilson $\longrightarrow$ continuous Hahn' and 'Askey-Wilson $\longrightarrow$ Jacobi' in Section 4 to the self-dual case (i.e. with Askey-Wilson parameters subject to the condition in (6.44a)), then we wind up with multivariable Wilson polynomials 
$p_{\lambda}^{W}(x)$ with parameters subject to the condition (cf. (4.6))

$$
\nu_{0}-\nu_{1}-\nu_{2}-\nu_{3}+1=0
$$

and with multivariable continuous Hahn polynomials $p_{\lambda}^{c H}(x)$ with parameters subject to the condition (cf. (4.10))

$$
\nu_{0}^{+}-\nu_{1}^{+}-\nu_{0}^{-}-\nu_{1}^{-}+1=0 .
$$

The (renormalized) Wilson type polynomials $P_{\lambda}^{W}(x)$ with parameters satisfying (6.44b) are again self-dual in the sense that for these parameters $\hat{\nu}_{r}=\nu_{r}(r=$ $0, \ldots, 3)$ and hence $\hat{P}_{\lambda}^{W}(x)=P_{\lambda}^{W}(x)$ (cf. Remark $i$. above). In the situation of a transition to the multivariable Jacobi polynomials, the restriction to the self-dual case does not lead to any conditions on the resulting Jacobi parameters $\nu_{0}, \nu_{1}$. The point is that if we substitute the Askey-Wilson parameters in accordance with (4.13), then imposing the self-duality condition (6.44a) gives rise to the condition $g_{0}-g_{0}^{\prime}-g_{1}-g_{1}^{\prime}=0$ on the parameters $g_{0}, g_{1}, g_{0}^{\prime}, g_{1}^{\prime}$. However, given this condition, the Jacobi parameters $\nu_{0}=g_{0}+g_{0}^{\prime}$ and $\nu_{1}=g_{1}+g_{1}^{\prime}$ (cf. Proposition 4.3) can still take arbitrary values. In other words: the confluence of the parameters in the limit $q \rightarrow 1$ has the consequence that the full three-parameter family of multivariable Jacobi type polynomials (parametrized by $\nu, \nu_{0}$ and $\nu_{1}$ ) is recovered as a limiting case of the self-dual multivariable Askey-Wilson polynomials with parameters satisfying (6.44a).

$i v$. The proof of the recurrence relations (Theorem 6.1 ), the duality relation (6.42a) and the evaluation/specialization formula (6.43a) for the multivariable Askey-Wilson polynomials presented in [D5] is complete only for parameters satisfying the self-duality condition in (6.44a). It is possible to extend the proof to the case of general Askey-Wilson parameters by invoking recent results due to Macdonald (see the note added in proof at the end of [D5], and cf. also the announcements at the end of [M5]). In view of the previous remark, the statements in Section 6 (and also Section 7) pertaining to the multivariable Wilson and continuous Hahn families thus also follow initially only for the restricted parameter domains subject to the additional constraints $(6.44 \mathrm{~b})$ and $(6.44 \mathrm{c})$, respectively. For the generalization to the case of general parameters, one has at this point to rely on the extension of the results on the multivariable Askey-Wilson polynomials of [D5] to the case of not necessarily self-dual parameters made possible by Macdonald's work. For the multivariable Jacobi family it is not necessary to invoke such an extended version of the results in [D5], since these polynomials may already be recovered fully from the self-dual Askey-Wilson family (see Remark iii., above).

\section{ORTHOGONALITY AND NORMALIZATION}

Note: In this section (except for Theorem 7.1, Proposition 7.2 and Gustafson's formulas in the remark at the end of the section and equations (7.26a)-(7.26c)) we assume Askey-Wilson, Wilson and continuous Hahn parameters subject to the constraints $q t_{0} t_{1}^{-1} t_{2}^{-1} t_{3}^{-1}=1,1+\nu_{0}-\nu_{1}-\nu_{2}-\nu_{3}=0$ and $1+\nu_{0}^{+}-\nu_{0}^{-}-\nu_{1}^{+}-\nu_{1}^{-}=0$, respectively. It is possible to extend the results of this section to general parameters by invoking a recent result due to Macdonald (see Remark $i v$. of Section 6).

Let us recall that the difference/differential equations in Section 5 hold for generic complex parameter values in view of the rational dependence on the parameters (cf. also Remark ii. of Section 4) and that the same is true for the recurrence relations in Section 6 (but keep in mind the note at the beginning of the section). In order to 
interpret the polynomials as an orthogonal system with weight function $\Delta$, however, rather than allowing generic complex parameters we will from now on always choose the parameters from the domains given in Section 2. (Recall that the conditions on the parameters in Section 2 ensure that the relevant weight functions $\Delta(x)$ are positive and that the integrals defining the associated inner products $\langle\cdot, \cdot\rangle_{\Delta}$ converge in absolute value; cf. Remark $i$. at the end of Section 2.)

It is immediate from the definition in Section 2 that the polynomial $p_{\lambda}$ is orthogonal to $p_{\mu}$ for $\mu<\lambda$. It turns out that also for weights that are not comparable with respect to the partial order (2.3), the associated polynomials are orthogonal.

Theorem 7.1 (Orthogonality). The multivariable Askey-Wilson, Wilson, continuous Hahn and Jacobi type polynomials $p_{\lambda}, \lambda \in \Lambda$, form an orthogonal system with respect to the $L^{2}$ inner product with weight function $\Delta(x)$, i.e.

$$
\left\langle p_{\lambda}, p_{\mu}\right\rangle_{\Delta}=0 \quad \text { if } \lambda \neq \mu
$$

(for parameters with values in the domains given in Section 2).

The proof of this theorem hinges on the following proposition.

Proposition 7.2 (Symmetry). The operators $D_{1}, \ldots, D_{n}$ of Section 5 are symmetric with respect to the inner product $\langle\cdot, \cdot\rangle_{\Delta}$, i.e.

$$
\left\langle D_{r} m_{\lambda}, m_{\mu}\right\rangle_{\Delta}=\left\langle m_{\lambda}, D_{r} m_{\mu}\right\rangle_{\Delta}
$$

(for parameters with values in the domains given in Section 2).

A detailed proof of the symmetry for the Askey-Wilson type difference operators can be found in [D1, Sec. 3.4]. The same reasoning used there can also be applied to prove the proposition for the Wilson and continuous Hahn case. The Jacobi case follows from the Askey-Wilson case with the aid of the limit transition from Askey-Wilson type to Jacobi type polynomials and operators (we refer to [D1, Sec. 4] for the precise details).

By combining Proposition 7.2 with the results of Section 5 we conclude that the polynomials $p_{\lambda}, \lambda \in \Lambda$, are joint eigenfunctions of $n$ independent operators $D_{1}, \ldots, D_{n}$ that are symmetric with respect to $\langle\cdot, \cdot\rangle_{\Delta}$. The corresponding (real) eigenvalues $E_{1, \lambda}, \ldots, E_{n, \lambda}$ separate the points of the integral cone $\Lambda$ (2.2), i.e., if $E_{r, \lambda}=E_{r, \mu}$ for $r=1, \ldots, n$ then $\lambda$ must be equal to $\mu$ (this is seen using the fact that the functions $E_{r}\left(x_{1}, \ldots, x_{n} ; y_{r}, \ldots y_{n}\right)$ (5.6) generate the algebra of permutation symmetric polynomials in the variables $\left.x_{1}, \ldots, x_{n}\right)$. It thus follows that the polynomials $p_{\lambda}$ and $p_{\mu}$ with $\lambda \neq \mu$ are orthogonal with respect to the inner product $\langle\cdot, \cdot\rangle_{\Delta}$ as eigenfunctions of a symmetric operator corresponding to different eigenvalues. In the case of Jacobi type polynomials, orthogonality proofs based on the simultaneous diagonalization by the algebra of commuting differential operators (not using the fact that these differential operators are a degeneration of the Askey-Wilson type difference operators) may be found in [De] and in [H, HS] (upon specialization to the root system $B C_{n}$ ). For the multivariable AskeyWilson case the original orthogonality proof by Macdonald [M2] and (in general) Koornwinder [K2] uses only the operator $D^{A W}$ (3.4) and the fact that its eigenvalues $E_{\lambda}^{A W}$ (3.7) are nondegenerate as functions of $q$ (Macdonald) or $t, t_{r}$ (Koornwinder), combined with the continuity of the innerproduct $\langle\cdot, \cdot\rangle_{\triangle}{ }^{A W}$ (2.5) with respect to these parameters.

The main purpose of this section is to express the squared norms of the polynomials (viz. $\left\langle p_{\lambda}, p_{\lambda}\right\rangle_{\Delta}$ ) in terms of the squared norm of the unit polynomial 
(viz. $\langle 1,1\rangle_{\Delta}$, which corresponds to $\lambda=0$ ) using a technique due to Koornwinder, who applied it to the case of type $A$ Macdonald polynomials (or multivariable $q$ ultraspherical polynomials) [K1, M4]. Our main tool to achieve this goal consists of the recurrence relations derived in the preceding section. Our starting point is the identity

$$
\left\langle\hat{E}_{r} P_{\lambda}, P_{\lambda+\omega_{r}}\right\rangle_{\Delta}=\left\langle P_{\lambda}, \hat{E}_{r} P_{\lambda+\omega_{r}}\right\rangle_{\Delta}, \quad \omega_{r}=e_{1}+\cdots+e_{r},
$$

where $P_{\lambda}(x)$ denotes the renormalized Askey-Wilson, Wilson, continuous Hahn or Jacobi type polynomial of the form (6.1) and $\hat{E}_{r}(x)$ is the corresponding symmetric function multiplying $P_{\lambda}(x)$ at the l.h.s. of the recurrence relation (6.3). (In all four cases of interest the functions $\hat{E}_{r}(x)$ are real for parameters in the domains of Section 2, and we hence have (7.3) trivially.) If we work out both sides of (7.3) by replacing $\hat{E}_{r} P_{\lambda}$ and $\hat{E}_{r} P_{\lambda+\omega_{r}}$ by the corresponding r.h.s. of (6.3) and use the orthogonality of the polynomials (Theorem 7.1), then we arrive at the following relation between $\left\langle P_{\lambda}, P_{\lambda}\right\rangle_{\Delta}$ and $\left\langle P_{\lambda+\omega_{r}}, p_{\lambda+\omega_{r}}\right\rangle_{\Delta}$ :

$$
\begin{aligned}
\hat{V}_{\{1, \ldots, r\},\{r+1, \ldots, n\}}(\rho+\lambda)\left\langle P_{\lambda+\omega_{r}}, P_{\lambda+\omega_{r}}\right\rangle_{\Delta} & \\
& =\hat{V}_{\{1, \ldots, r\},\{r+1, \ldots, n\}}\left(-\rho-\lambda-\omega_{r}\right)\left\langle P_{\lambda}, P_{\lambda}\right\rangle_{\Delta} .
\end{aligned}
$$

(Recall that $\hat{U}_{K, p}=1$ for $p=0$ and that $\hat{V}_{\{1, \ldots, r\},\{r+1, \ldots, n\}}$ is taken to be in accordance with the definition in (6.3) with all signs $\varepsilon_{j}, j \in J$, being positive.) In principle one can use this relation to obtain for each $\lambda \in \Lambda$ the squared norm of $P_{\lambda}(x)$ in terms of the squared norm of $P_{0}(x)(=1)$ by writing $\lambda$ as a positive linear combination of the (fundamental weight) vectors $\omega_{1}, \ldots, \omega_{n}$ and then applying (7.4) iteratively by walking to the weight $\lambda$ starting from the zero weight $(0, \ldots, 0)$ through the successive addition of fundamental weight vectors $\omega_{r}$. Indeed, it is not difficult to verify that the combinatorial structure of the coefficients $\hat{V}_{\{1, \ldots, r\},\{r+1, \ldots, n\}}$ is such that the result does not depend on the order in which the fundamental weight vectors $\omega_{r}$ are added, i.e., the result is independent of the chosen path from $(0, \ldots, 0)$ to $\lambda$. This hinges on the easily inferred (combinatorial) identity

$$
\begin{aligned}
& \frac{\hat{V}_{\{1, \ldots, r\},\{r+1, \ldots, n\}}\left(-x-\omega_{s}-\omega_{r}\right)}{\hat{V}_{\{1, \ldots, r\},\{r+1, \ldots, n\}}\left(x+\omega_{s}\right)} \frac{\hat{V}_{\{1, \ldots, s\},\{s+1, \ldots, n\}}\left(-x-\omega_{s}\right)}{\hat{V}_{\{1, \ldots, s\},\{s+1, \ldots, n\}}(x)} \\
& =\frac{\hat{V}_{\{1, \ldots, s\},\{s+1, \ldots, n\}}\left(-x-\omega_{r}-\omega_{s}\right)}{\hat{V}_{\{1, \ldots, s\},\{s+1, \ldots, n\}}\left(x+\omega_{r}\right)} \frac{\hat{V}_{\{1, \ldots, r\},\{r+1, \ldots, n\}}\left(-x-\omega_{r}\right)}{\hat{V}_{\{1, \ldots, r\},\{r+1, \ldots, n\}}(x)},
\end{aligned}
$$

which expresses the fact that the result for the quotient of $\left\langle P_{\lambda+\omega_{r}+\omega_{s}}, P_{\lambda+\omega_{r}+\omega_{s}}\right\rangle_{\Delta}$ and $\left\langle P_{\lambda}, P_{\lambda}\right\rangle_{\Delta}$ computed via (7.4) does not depend on the order in which $\omega_{r}$ and $\omega_{s}$ are added (as it clearly should not).

To write down the answer for $\left\langle P_{\lambda}, P_{\lambda}\right\rangle_{\Delta} /\langle 1,1\rangle_{\Delta}$ for general $\lambda \in \Lambda$, we introduce the functions

$$
\hat{\Delta}_{ \pm}(x)=\prod_{1 \leq j<k \leq n} \hat{d}_{v, \pm}\left(x_{j}+x_{k}\right) \hat{d}_{v, \pm}\left(x_{j}-x_{k}\right) \prod_{1 \leq j \leq n} \hat{d}_{w, \pm}\left(x_{j}\right),
$$

with $\hat{d}_{v, \pm}(z)$ and $\hat{d}_{w, \pm}(z)(\neq 0)$ satisfying the difference equations

$$
\begin{aligned}
\hat{d}_{v,+}(z+1) & =\hat{v}(z) \hat{d}_{v,+}(z), & \hat{d}_{v,-}(z+1) & =\hat{v}(-z-1) \hat{d}_{v,-}(z), \\
\hat{d}_{w,+}(z+1) & =\hat{w}(z) \hat{d}_{w,+}(z), & \hat{d}_{w,-}(z+1) & =\hat{w}(-z-1) \hat{d}_{w,-}(z),
\end{aligned}
$$


where $\hat{v}(z)$ and $\hat{w}(z)$ are taken to be the same as in Section 6. It is immediate from the difference equations (7.6), (7.7) that

$$
\begin{aligned}
& \frac{\hat{\Delta}_{+}\left(x+\omega_{r}\right)}{\hat{\Delta}_{+}(x)}=\hat{V}_{\{1, \ldots, r\},\{r+1, \ldots, n\}}(x), \\
& \frac{\hat{\Delta}_{-}\left(x+\omega_{r}\right)}{\hat{\Delta}_{-}(x)}=\hat{V}_{\{1, \ldots, r\},\{r+1, \ldots, n\}}\left(-x-\omega_{r}\right) .
\end{aligned}
$$

With the aid of the properties (7.8) and (7.9) we can rewrite (7.4) in the form

$$
\left\langle P_{\lambda}, P_{\lambda}\right\rangle_{\Delta} \frac{\hat{\Delta}_{+}(\rho+\lambda)}{\hat{\Delta}_{-}(\rho+\lambda)}=\left\langle P_{\lambda+\omega_{r}}, P_{\lambda+\omega_{r}}\right\rangle_{\Delta} \frac{\hat{\Delta}_{+}\left(\rho+\lambda+\omega_{r}\right)}{\hat{\Delta}_{-}\left(\rho+\lambda+\omega_{r}\right)} .
$$

By using the fact that the fundamental weight vectors $\omega_{1}, \ldots, \omega_{n}$ positively generate the integral cone $\Lambda$ (2.2), one deduces from this equation that the quotient $\left\langle P_{\lambda}, P_{\lambda}\right\rangle_{\Delta} \hat{\Delta}_{+}(\rho+\lambda) / \hat{\Delta}_{-}(\rho+\lambda)$ in the l.h.s. of (7.10) does not depend on the choice of $\lambda \in \Lambda$. Comparing with its evaluation at $\lambda=0$ then entails

$$
\frac{\left\langle P_{\lambda}, P_{\lambda}\right\rangle_{\Delta}}{\langle 1,1\rangle_{\Delta}}=\frac{\hat{\Delta}_{-}(\rho+\lambda) \hat{\Delta}_{+}(\rho)}{\hat{\Delta}_{+}(\rho+\lambda) \hat{\Delta}_{-}(\rho)} .
$$

As we will see below (and is suggested by the notation), it turns out that the function $\Delta_{+}(x)$ in the present section coincides with that of Section 6 . So, by combining (7.11) with (6.1) we obtain

$$
\frac{\left\langle p_{\lambda}, p_{\lambda}\right\rangle_{\Delta}}{\langle 1,1\rangle_{\Delta}}=|c|^{-2|\lambda|} \frac{\hat{\Delta}_{+}(\rho+\lambda) \hat{\Delta}_{-}(\rho+\lambda)}{\hat{\Delta}_{+}(\rho) \hat{\Delta}_{-}(\rho)} .
$$

We will now list for each of our four families $\mathrm{AW}, \mathrm{W}, \mathrm{cH}$ and $\mathrm{J}$ the associated functions $\hat{d}_{v, \pm}(z)$ and $\hat{d}_{w, \pm}(z)$, and formulate the corresponding evaluation theorem for the quotient of $\left\langle p_{\lambda}, p_{\lambda}\right\rangle_{\Delta}$ and $\langle 1,1\rangle_{\Delta}$. The proof of this theorem in each case boils down to verifying that $\hat{d}_{v, \pm}(z)$ and $\hat{d}_{w, \pm}(z)$ indeed satisfy the difference equations (7.6) and (7.7).

For the multivariable Jacobi polynomials the value of the integral for the squared norm $\left\langle p_{\lambda}, p_{\lambda}\right\rangle_{\Delta}$ was first computed by Opdam [Op1] with the aid of shift operators (see also [HS]). It turned out that one can simplify Opdam's shift-operator method somewhat with the aid of an extension to the case of nonsymmetric polynomials [Op2] (see also [M5, C5] for the extension to the $q$-case) based (a.o.) on the ideas of Dunkl [Du1, Du2] combined with (graded) Hecke-algebraic techniques due to Cherednik [C1, C2]. In this special case our proof of the norm formulas by means of recurrence relations rather than shift operators provides an alternative approach towards this question.

The expressions for the squared norms of the multivariable Askey-Wilson polynomials were conjectured by Macdonald [M2, M3] and then proven by Cherednik [C3] for special parameters related to the reduced root systems (and admissible pairs of the form $\left(R, R^{\vee}\right)$ ) using a generalization of Opdam's shift operator approach (see also [C4]). Recently, Macdonald announced a further extension of these methods to the case of general Askey-Wilson parameters [M5]. For the Askey-Wilson type family, the above proof of the norm formulas based on the recurrence relations in combination with Gustafson's evaluation formula for the generalized Selberg integral [Gu1, Ka] was presented in [D5] (for parameters subject to the condition in 
the note at the beginning of the section). This case is reproduced here for the sake of completeness.

7.1. Askey-Wilson type. In the case of Askey-Wilson type polynomials the functions $\hat{\Delta}_{ \pm}(x)=\hat{\Delta}_{ \pm}^{A W}(x)$ of the form in (7.5) are characterized by $\hat{d}_{v,+}^{A W}(z)(6.6)$, $\hat{d}_{w,+}^{A W}(z)(6.7)$ and

$$
\begin{aligned}
& \hat{d}_{v,-}^{A W}(z)=t^{z / 2} \frac{\left(q^{z+1} ; q\right)_{\infty}}{\left(t^{-1} q^{z+1} ; q\right)_{\infty}} \\
& \hat{d}_{w,-}^{A W}(z)=\left(\hat{t}_{0} \hat{t}_{1} \hat{t}_{2} \hat{t}_{3} q^{-1}\right)^{z / 2} \frac{\left(q^{2 z+1} ; q\right)_{\infty}}{\left(\hat{t}_{0}^{-1} q^{z+1}, \hat{t}_{1}^{-1} q^{z+1}, \hat{t}_{2}^{-1} q^{z+1}, \hat{t}_{3}^{-1} q^{z+1} ; q\right)_{\infty}}
\end{aligned}
$$

(with $\hat{t}_{r}$ given by (6.8)). Formula (7.12) leads us to the following theorem for this case.

Theorem 7.3 ([D5]). For Askey-Wilson parameters with values taken from the domain indicated in Section 2.1, one has

$$
\frac{\left\langle p_{\lambda}^{A W}, p_{\lambda}^{A W}\right\rangle_{\Delta^{A W}}}{\langle 1,1\rangle_{\Delta^{A W}}}=\frac{\hat{\Delta}_{+}^{A W}\left(\rho^{A W}+\lambda\right) \hat{\Delta}_{-}^{A W}\left(\rho^{A W}+\lambda\right)}{\hat{\Delta}_{+}^{A W}\left(\rho^{A W}\right) \hat{\Delta}_{-}^{A W}\left(\rho^{A W}\right)},
$$

with $\hat{\Delta}_{ \pm}^{A W}(x)$ (7.5) determined by $\hat{d}_{v,+}^{A W}(6.6), \hat{d}_{w,+}^{A W}(6.7), \hat{d}_{v,-}^{A W}$ (7.13), $\hat{d}_{w,-}^{A W}$ (7.14), and $\rho^{A W}$ taken from (6.9).

To complete the proof of the theorem it suffices to infer that the functions $\hat{d}_{v, \pm}^{A W}(z)$ and $\hat{d}_{w, \pm}^{A W}(z)$ indeed satisfy the corresponding difference equations of the form in (7.6) and (7.7); a fact not difficult to deduce from the standard relation for the $q$-shifted factorials $(a ; q)_{\infty}=(1-a)(a q ; q)_{\infty}$.

For $n=1$ the r.h.s. of (7.15) reduces to an expression of the form

$$
\left\langle p_{l}^{A W}, p_{l}^{A W}\right\rangle_{\Delta^{A W}} /\left\langle p_{0}^{A W}, p_{0}^{A W}\right\rangle_{\Delta^{A W}}
$$

with (cf. $[\mathrm{AW} 2, \mathrm{KS}]$ and recall our normalization in $(2.8)$ )

$$
\left\langle p_{l}^{A W}, p_{l}^{A W}\right\rangle_{\Delta^{A W}}=\frac{2\left(t_{0} t_{1} t_{2} t_{3} q^{2 l} ; q\right)_{\infty}}{\left(t_{0} t_{1} t_{2} t_{3} q^{l-1} ; q\right)_{l}\left(q^{l+1} ; q\right)_{\infty} \prod_{0 \leq r<s \leq 3}\left(t_{r} t_{s} q^{l} ; q\right)_{\infty}} .
$$

7.2. Wilson type. The relevant functions $\hat{\Delta}_{ \pm}(x)=\hat{\Delta}_{ \pm}^{W}(x)$ of the form in (7.5) are determined by $\hat{d}_{v,+}^{W}(z), \hat{d}_{w,+}^{W}(z)(6.19)$ and

$$
\hat{d}_{v,-}^{W}(z)=\frac{\Gamma(-\nu+z+1)}{\Gamma(z+1)}, \quad \hat{d}_{w,-}^{W}(z)=\frac{\prod_{0 \leq r \leq 3} \Gamma\left(-\hat{\nu}_{r}+z+1\right)}{\Gamma(2 z+1)}
$$

(with $\hat{\nu}_{r}$ given by (6.20)). The difference equations of the form in (7.6) and (7.7) are easily verified using the standard functional equation $\Gamma(z+1)=z \Gamma(z)$ for the gamma function. The normalization theorem becomes, in the present situation,

Theorem 7.4. For Wilson parameters with values taken from the domain indicated in Section 2.2, one has

$$
\frac{\left\langle p_{\lambda}^{W}, p_{\lambda}^{W}\right\rangle_{\Delta^{W}}}{\langle 1,1\rangle_{\Delta^{W}}}=\frac{\hat{\Delta}_{+}^{W}\left(\rho^{W}+\lambda\right) \hat{\Delta}_{-}^{W}\left(\rho^{W}+\lambda\right)}{\hat{\Delta}_{+}^{W}\left(\rho^{W}\right) \hat{\Delta}_{-}^{W}\left(\rho^{W}\right)}
$$

with $\hat{\Delta}_{ \pm}^{W}(x)$ (7.5) determined by $\hat{d}_{v,+}^{W}, \hat{d}_{w,+}^{W}$ (6.19) and $\hat{d}_{v,-}^{W}, \hat{d}_{w,-}^{W}(7.17)$, and $\rho^{W}$ taken from (5.12). 
For $n=1$ the r.h.s. of (7.18) reduces to

$$
\left\langle p_{l}^{W}, p_{l}^{W}\right\rangle_{\Delta W} /\left\langle p_{0}^{W}, p_{0}^{W}\right\rangle_{\Delta^{W}}
$$

with (cf. [W, KS] and recall our normalization in (2.13))

$$
\left\langle p_{l}^{W}, p_{l}^{W}\right\rangle_{\Delta W}=\frac{2 l ! \prod_{0 \leq r<s \leq 3} \Gamma\left(\nu_{r}+\nu_{s}+l\right)}{\left(\nu_{0}+\nu_{1}+\nu_{2}+\nu_{3}+l-1\right)_{l} \Gamma\left(\nu_{0}+\nu_{1}+\nu_{2}+\nu_{3}+2 l\right)} .
$$

7.3. Continuous Hahn type. The functions $\hat{\Delta}_{ \pm}(x)=\hat{\Delta}_{ \pm}^{c H}(x)$ of the form in (7.5) are now determined by $\hat{d}_{v,+}^{c H}(z), \hat{d}_{w,+}^{c H}(z)(6.27)$ and

$$
\hat{d}_{v,-}^{c H}(z)=\frac{\Gamma(-\nu+z+1)}{\Gamma(z+1)}, \quad \hat{d}_{w,-}^{c H}(z)=\frac{\prod_{0 \leq r \leq 2} \Gamma\left(-\hat{\nu}_{r}+z+1\right)}{\Gamma(2 z+1)}
$$

(with $\hat{\nu}_{r}$ taken from (6.28)). The difference equations (7.6), (7.7) follow again from the standard difference equation for the gamma function. The normalization theorem reads, in this case,

Theorem 7.5. For continuous Hahn parameters with values taken from the domain indicated in Section 2.3, one has

$$
\frac{\left\langle p_{\lambda}^{c H}, p_{\lambda}^{c H}\right\rangle_{\Delta^{c H}}}{\langle 1,1\rangle_{\Delta^{c H}}}=\frac{\hat{\Delta}_{+}^{c H}\left(\rho^{c H}+\lambda\right) \hat{\Delta}_{-}^{c H}\left(\rho^{c H}+\lambda\right)}{\hat{\Delta}_{+}^{c H}\left(\rho^{c H}\right) \hat{\Delta}_{-}^{c H}\left(\rho^{c H}\right)}
$$

with $\hat{\Delta}_{ \pm}^{c H}(x)(7.5)$ determined by $\hat{d}_{v,+}^{c H}, \hat{d}_{w,+}^{c H}(6.27)$ and $\hat{d}_{v,-}^{c H}, \hat{d}_{w,-}^{c H}(7.20)$, and $\rho^{c H}$ given by (5.18).

For $n=1$ the r.h.s. of $(7.21)$ becomes

$$
\left\langle p_{l}^{c H}, p_{l}^{c H}\right\rangle_{\Delta^{c H}} /\left\langle p_{0}^{c H}, p_{0}^{c H}\right\rangle_{\Delta^{c H}}
$$

with (cf. [AW1, AtSu, A, KS] and recall our normalization in (2.18))

$$
\begin{array}{rl}
\left\langle p_{l}^{c H}, p_{l}^{c H}\right\rangle_{\Delta^{c H}} & l ! \prod_{r, s \in\{0,1\}} \Gamma\left(\nu_{r}^{+}+\nu_{s}^{-}+l\right) \\
& =\frac{\left(\nu_{0}^{+}+\nu_{0}^{-}+\nu_{1}^{+}+\nu_{1}^{-}+l-1\right)_{l} \Gamma\left(\nu_{0}^{+}+\nu_{0}^{-}+\nu_{1}^{+}+\nu_{1}^{-}+2 l\right)}{} .
\end{array}
$$

7.4. Jacobi type. For the Jacobi type polynomials the functions $\hat{\Delta}_{ \pm}(x)=\hat{\Delta}_{ \pm}^{J}(x)$ of the form in (7.5) are determined by $\hat{d}_{v,+}^{J}(z), \hat{d}_{w,+}^{J}(z)(6.35)$ and

$$
\hat{d}_{v,-}^{J}(z)=\frac{\Gamma(-\nu+z+1)}{\Gamma(z+1)}, \quad \hat{d}_{w,-}^{J}(z)=\frac{\Gamma\left(-\hat{\nu}_{0}+z+1\right) \Gamma\left(-\hat{\nu}_{1}+z+1\right)}{\Gamma(2 z+1)}
$$

(with $\hat{\nu}_{0}, \hat{\nu}_{1}$ taken from (6.36)). The difference equations (7.6), (7.7) follow again from the difference equation for the gamma function. The normalization theorem reads, in this case,

Theorem 7.6 ([Op1, Op2, HS]). For Jacobi parameters with values taken from the domain indicated in Section 2.4, one has

$$
\frac{\left\langle p_{\lambda}^{J}, p_{\lambda}^{J}\right\rangle_{\Delta^{J}}}{\langle 1,1\rangle_{\Delta^{J}}}=2^{4|\lambda|} \frac{\hat{\Delta}_{+}^{J}\left(\rho^{J}+\lambda\right) \hat{\Delta}_{-}^{J}\left(\rho^{J}+\lambda\right)}{\hat{\Delta}_{+}^{J}\left(\rho^{J}\right) \hat{\Delta}_{-}^{J}\left(\rho^{J}\right)}
$$

with $\hat{\Delta}_{ \pm}^{J}(x)$ (7.5) determined by $\hat{d}_{v,+}^{J}, \hat{d}_{w,+}^{J}$ (6.35) and $\hat{d}_{v,-}^{J}, \hat{d}_{w,-}^{J}$ (7.23), and $\rho^{J}$ given by (5.21)). 
For $n=1$ the r.h.s. of (7.24) becomes

$$
\left\langle p_{l}^{J}, p_{l}^{J}\right\rangle_{\Delta^{J}} /\left\langle p_{0}^{J}, p_{0}^{J}\right\rangle_{\Delta^{J}}
$$

with (cf. [AbSt, KS], and recall our normalization in (2.21))

$$
\left\langle p_{l}^{J}, p_{l}^{J}\right\rangle_{\Delta^{J}}=\frac{2^{4 l} \pi^{-1} l ! \Gamma\left(\nu_{0}+1 / 2+l\right) \Gamma\left(\nu_{1}+1 / 2+l\right)}{\left(\nu_{0}+\nu_{1}+l\right)_{l} \Gamma\left(\nu_{0}+\nu_{1}+2 l+1\right)} .
$$

Remark. The integral of the weight function for the multivariable Jacobi polynomials boils down to an integral evaluated by Selberg [M1, Se]. The corresponding integrals for the multivariable Askey-Wilson, Wilson and continuous Hahn polynomials amount to certain generalizations of the Selberg integral found by Gustafson [Gu1] (for the Askey-Wilson case see also Kadell [Ka]). Explicitly, one has [Gu1, Ka, M1, Se]:

$$
\begin{aligned}
\langle 1,1\rangle_{\Delta^{A W}} & =2^{n} n ! \prod_{1 \leq j \leq n} \frac{(t ; q)_{\infty}\left(t_{0} t_{1} t_{2} t_{3} t^{n+j-2} ; q\right)_{\infty}}{(q, q)_{\infty}\left(t^{j} ; q\right)_{\infty} \prod_{0 \leq r<s \leq 3}\left(t_{r} t_{s} t^{j-1} ; q\right)_{\infty}}, \\
\langle 1,1\rangle_{\Delta^{W}} & =2^{n} n ! \prod_{1 \leq j \leq n} \frac{\Gamma(\nu j) \prod_{0 \leq r<s \leq 3} \Gamma\left(\nu_{r}+\nu_{s}+(j-1) \nu\right)}{\Gamma(\nu) \Gamma\left(\nu_{0}+\nu_{1}+\nu_{2}+\nu_{3}+(n+j-2) \nu\right)}, \\
\langle 1,1\rangle_{\Delta^{c H}} & =n ! \prod_{1 \leq j \leq n} \frac{\Gamma(\nu j) \prod_{r, s \in\{0,1\}} \Gamma\left(\nu_{r}^{+}+\nu_{s}^{-}+(j-1) \nu\right)}{\Gamma(\nu) \Gamma\left(\nu_{0}^{+}+\nu_{0}^{-}+\nu_{1}^{+}+\nu_{1}^{-}+(n+j-2) \nu\right)}, \\
\langle 1,1\rangle_{\Delta^{J}} & =\frac{n !}{\pi^{n}} \prod_{1 \leq j \leq n} \frac{\Gamma(\nu j) \Gamma\left(1 / 2+\nu_{0}+(j-1) \nu\right) \Gamma\left(1 / 2+\nu_{1}+(j-1) \nu\right)}{\Gamma(\nu) \Gamma\left(1+\nu_{0}+\nu_{1}+(n+j-2) \nu\right)} .
\end{aligned}
$$

In [Gu1] the proof of the integration formula for $\langle 1,1\rangle_{\Delta^{A W}}$ is given, but that of $\langle 1,1\rangle_{\Delta^{W}}$ and $\langle 1,1\rangle_{\Delta^{c H}}$ is omitted. One readily constructs such proofs oneself, however, by mimicking Gustafson's proof for the multivariable Askey-Wilson integral $\langle 1,1\rangle_{\triangle A W}$ starting from the integration formulas in Theorem 5.1 (for the continuous Hahn case) and Theorem 9.3 (for the Wilson case) of [Gu2].

It is moreover not very difficult to verify that the above evaluation formulas for the integral $\langle 1,1\rangle_{\Delta}$ can be rewritten in the form

$$
\begin{aligned}
\langle 1,1\rangle_{\Delta^{A W}} & =2^{n} n ! \hat{\Delta}_{+}^{A W}\left(\rho^{A W}\right) \hat{\Delta}_{-}^{A W}\left(\rho^{A W}\right), \\
\langle 1,1\rangle_{\Delta^{W}} & =2^{n} n ! \hat{\Delta}_{+}^{W}\left(\rho^{W}\right) \hat{\Delta}_{-}^{W}\left(\rho^{W}\right), \\
\langle 1,1\rangle_{\Delta^{c H}} & =n ! \hat{\Delta}_{+}^{c H}\left(\rho^{c H}\right) \hat{\Delta}_{-}^{c H}\left(\rho^{c H}\right), \\
\langle 1,1\rangle_{\Delta^{J}} & =\pi^{-n} n ! \hat{\Delta}_{+}^{J}\left(\rho^{J}\right) \hat{\Delta}_{-}^{J}\left(\rho^{J}\right),
\end{aligned}
$$

which combines with the expressions for the ratios $\left\langle p_{\lambda}, p_{\lambda}\right\rangle_{\Delta} /\langle 1,1\rangle_{\Delta}$ given by Theorems 7.3-7.6 into the following evaluation formulas for the squared norms of the polynomials $p_{\lambda}(x)$ (cf. [Op1, Op2, HS] for the Jacobi case and [M2, M3, C3, C4, M5, D5] for the Askey-Wilson case):

$$
\begin{aligned}
\left\langle p_{\lambda}^{A W}, p_{\lambda}^{A W}\right\rangle_{\Delta^{A W}} & =2^{n} n ! \hat{\Delta}_{+}^{A W}\left(\rho^{A W}+\lambda\right) \hat{\Delta}_{-}^{A W}\left(\rho^{A W}+\lambda\right), \\
\left\langle p_{\lambda}^{W}, p_{\lambda}^{W}\right\rangle_{\Delta^{W}} & =2^{n} n ! \hat{\Delta}_{+}^{W}\left(\rho^{W}+\lambda\right) \hat{\Delta}_{-}^{W}\left(\rho^{W}+\lambda\right), \\
\left\langle p_{\lambda}^{c H}, p_{\lambda}^{c H}\right\rangle_{\Delta^{c H}} & =n ! \hat{\Delta}_{+}^{c H}\left(\rho^{c H}+\lambda\right) \hat{\Delta}_{-}^{c H}\left(\rho^{c H}+\lambda\right), \\
\left\langle p_{\lambda}^{J}, p_{\lambda}^{J}\right\rangle_{\Delta^{J}} & =2^{4|\lambda|} \pi^{-n} n ! \hat{\Delta}_{+}^{J}\left(\rho^{J}+\lambda\right) \hat{\Delta}_{-}^{J}\left(\rho^{J}+\lambda\right) .
\end{aligned}
$$




\section{ACKNOWLEDGMENTS}

The author would like to thank Professor T. Oshima for kind hospitality at the University of Tokyo. Thanks are also due to J. V. Stokman for pointing out the usefulness of formula (4.1) in studying the transition to degenerate cases of the multivariable Askey-Wilson polynomials, and to the referee for suggesting some improvements.

\section{REFERENCES}

[AbSt] M. Abramowitz and I. A. Stegun (eds.), Handbook of mathematical functions, Dover Publications, New York, 1972 (9th printing). MR 85j:00005a; MR 94b:00012 (reprints)

[A] R. Askey, Continuous Hahn polynomials, J. Phys. A: Math. Gen. 18 (1985), L1017-1019. MR 87d:33021

[AW1] R. Askey and J. Wilson, A set of hypergeometric orthogonal polynomials, SIAM J. Math. Anal. 13 (1982), 651-655. MR 83h:33010

[AW2] _ Some basic hypergeometric orthogonal polynomials that generalize Jacobi polynomials, Mem. Amer. Math. Soc. 54 (1985), no. 319. MR 87a:05023

[AtSu] N. M. Atakishiyev and S. K. Suslov, The Hahn and Meixner polynomials of an imaginary argument and some of their applications, J. Phys. A: Math. Gen. 18 (1985), 1583-1596. MR 87i: 33021

[BO] R. J. Beerends and E. M. Opdam, Certain hypergeometric series related to the root system $B C$, Trans. Amer. Math. Soc. 339 (1993), 581-609. MR 94e:33024

[C1] I. Cherednik, A unification of Knizhnik-Zamolodchikov and Dunkl operators via affine Hecke algebras, Invent. Math. 106 (1991), 411-431. MR 93b:17040

[C2] - Integration of quantum many-body problems by affine Knizhnik-Zamolodchikov equations, Adv. Math. 106 (1994), 65-95. MR 95m:32021

[C3] , Double affine Hecke algebras and Macdonald's conjectures, Ann. Math. 141 (1995), 191-216. MR 96m:33010

[C4] , Macdonald's evaluation conjectures and difference Fourier transform, Invent. Math. 122 (1995), 119-145; 125 (1996), 391. CMP 96:02,14

[C5] , Nonsymmetric Macdonald polynomials, Internat. Math. Res. Notices 3 (1995), 483-515. MR 97f:33032

[De] A. Debiard, Système différentiel hypergéométrique et parties radiales des opérateurs invariants des espaces symétriques de type $B C_{p}$, in: Séminaire d'Algèbre Paul Dubreil et Marie-Paule Malliavin (M.-P. Malliavin, ed.), Lecture Notes in Math., vol. 1296, Springer, Berlin, 1988, pp. 42-124. MR 89e:22027

[D1] J. F. van Diejen, Commuting difference operators with polynomial eigenfunctions, Compositio Math. 95 (1995), 183-233. MR 96i:39023

[D2] , Difference Calogero-Moser systems and finite Toda chains, J. Math. Phys. 36 (1995), 1299-1323. MR 96d:70014

[D3] Multivariable continuous Hahn and Wilson polynomials related to integrable difference systems, J. Phys. A: Math. Gen. 28 (1995), L369-L374. MR 96i:33026

[D4] On the diagonalization of difference Calogero-Sutherland systems, in: Symmetries and integrability of difference equations (D. Levi, L. Vinet, and P. Winternitz, eds.), CRM Proceedings and Lecture Notes, vol. 9, Amer. Math. Soc., Providence, R.I., 1996, pp. 79-89. CMP 97:03

[D5] _ Self-dual Koornwinder-Macdonald polynomials, Invent. Math. 126 (1996), 319339. CMP 97:02

[Du1] C. F. Dunkl, Reflection groups and orthogonal polynomials on the sphere, Math. Z. 197 (1988), 33-60. MR 89b:42016

[Du2] , Differential-difference operators associated to reflection groups, Trans. Amer. Math. Soc. 311 (1989), 167-183. MR 90k:33027

[GR] G. Gasper and M. Rahman, Basic hypergeometric series, Cambridge University Press, Cambridge, 1990. MR 91d:33034

[Gu1] R. A. Gustafson, A generalization of Selberg's beta integral, Bull. Amer. Math. Soc. (N.S.) 22 (1990), 97-105. MR 90j:33001 
[Gu2] _ Some q-beta and Mellin-Barnes integrals on compact Lie groups and Lie algebras, Trans. Amer. Math. Soc. 341 (1994), 69-119. MR 94c:33032

[H] G. J. Heckman, An elementary approach to the hypergeometric shift operator of Opdam, Invent. Math. 103 (1991), 341-350. MR 92i:33012

[HS] G. J. Heckman and H. Schlichtkrull, Harmonic analysis and special functions on symmetric spaces, Perspectives in Math., vol. 16, Academic Press, San Diego, 1994. MR 96j:22019

[Ka] K. W. J. Kadell, A proof of the q-Macdonald-Morris conjecture for $B C_{n}$, Mem. Amer. Math. Soc. 108 (1994), no. 516. MR 94h:33013

[KS] R. Koekoek and R. F. Swarttouw, The Askey-scheme of hypergeometric orthogonal polynomials and its q-analogue, Math. report Delft Univ. of Technology 94-05, 1994.

[K1] T. H. Koornwinder, Self-duality for q-ultraspherical polynomials associated with the root system $A_{n}$, unpublished manuscript, 1988.

[K2] - Askey-Wilson polynomials for root systems of type BC, in: Hypergeometric functions on domains of positivity, Jack polynomials, and applications (D. St. P. Richards, ed.), Contemp. Math., vol. 138, Amer. Math. Soc., Providence, R. I., 1992, pp. 189-204. MR 94e:33039

[M1] I. G. Macdonald, Some conjectures for root systems, SIAM J. Math. Anal. 13 (1982), 988-1007. MR 84h:17006a

[M2] , Orthogonal polynomials associated with root systems, unpublished manuscript, 1988. See MR 91k:00039

[M3] Some conjectures for Koornwinder's orthogonal polynomials, unpublished manuscript, 1991.

[M4] Symmetric functions and Hall polynomials, 2nd edition, Clarendon Press, Oxford, 1995. MR 96h:05027

[M5] Affine Hecke algebras and orthogonal polynomials, Séminaire Bourbaki 1994/95, Astérisque No. 237 (1996), pp. 189-207. CMP 97:05

[OOS] H. Ochiai, T. Oshima, and H. Sekiguchi, Commuting families of symmetric differential operators, Proc. Japan Acad. Ser. A Math. Sci. 70 (1994), 62-66. CMP 94:11

[OP] M. A. Olshanetsky and A. M. Perelomov, Quantum integrable systems related to Lie algebras, Phys. Reps. 94 (1983), 313-404. MR 84k:81007

[Op1] E. M. Opdam, Some applications of hypergeometric shift operators, Invent. Math. 98 (1989), 1-18. MR 91h:33024

[Op2] Harmonic analysis for certain representations of graded Hecke algebras, Acta Math. 175 (1993), 75-121. CMP 96:01

[OS] T. Oshima and H. Sekiguchi, Commuting families of differential operators invariant under the action of a Weyl group, J. Math. Sci. Univ. Tokyo 2 (1995), 1-75. MR 96k:35006

[R1] S. N. M. Ruijsenaars, Complete integrability of relativistic Calogero-Moser systems and elliptic function identities, Commun. Math. Phys. 110 (1987), 191-213. MR 88i:58072

[R2] - Finite-dimensional soliton systems. in.: Integrable and superintegrable systems (B. Kupershmidt, ed.), World Scientific, Singapore, 1990, pp. 165-206. MR 92g:58056

[Se] A. Selberg, Bemerkninger om et multipelt integral, Norsk Mat. Tidsskr. 26 (1944), 71-78 (Collected papers, vol. 1, Springer, Berlin, 1989, pp. 204-213). MR 8:269b; MR 92h:01083

[S1] J. V. Stokman, Multivariable big and little q-Jacobi polynomials, SIAM J. Math. Anal. 28 (1997), 452-480. CMP 97:08

[S2] Multivariable BC type Askey-Wilson polynomials with partly discrete orthogonality measure, Ramanujan J. 1 (1997), 275-297. CMP 98:08

[SK] J. V. Stokman and T. H. Koornwinder, Limit transitions for BC type multivariable orthogonal polynomials, Canad. J. Math. 49 (1997), 373-404. CMP 97:12

[V] L. Vretare, Formulas for elementary spherical functions and generalized Jacobi polynomials, SIAM J. Math. Anal. 15 (1984), 805-833. MR 86k:33018

[W] J. A. Wilson, Some hypergeometric orthogonal polynomials, SIAM J. Math. Anal. 11 (1980), 690-701. MR 82a:33014

Department of Mathematical Sciences, The University of Tokyo, 3-8-1 Komaba, Meguro-Ku, TOKYO 153, JAPAN

Current address: Departamento de Matemáticas, Facultad de Ciencias, Universidad de Chile, Casilla 653, Santiago 1, Chile 